\title{
Cohabitation versus marriage: Marriage matching with peer effects
}

\author{
Ismael Mourifié and Aloysius Siow* \\ University of Toronto
}

November 24, 2014

\begin{abstract}
This paper proposes an easy to estimate Cobb Douglas marriage matching function (MMF). Special cases include the Choo Siow (CS) MMF, CS with peer effects, CS with frictional transfers, the Dagsvik Menzel nontransferable utility MMF and Chiappori, Salanié and Weiss MMF. Given population supplies and admissible parameters, the Cobb Douglas MMF exists and is unique. This MMF is estimated on US marriage and cohabitation data by states from 1990 to 2010. CS with peer effects is not rejected. There are peer and scale effects in the US marriage markets. Positive assortative matching in marriage and cohabitation by educational attainment are relatively stable from 1990 to 2010.
\end{abstract}

Since the seventies, marital behavior in the United States have changed significantly. First, for most adult groups, marriage rates have fallen. Second, starting from a very low initial rate, cohabitation rates have risen significantly. Because the initial cohabitation rates were so low, the rise in cohabitations did not compensate for the fall in marriages. So third, the fraction of adults who are unmatched, i.e. not married or cohabitating, have risen significantly. Evidence for these trends for women and men between ages 26-30 and 28-32 respectively are shown in Figure 1 in Appendix C. ${ }^{1}$

Researchers have investigated different causes for these changes including changes in reproductive technologies as well as access to them, changes in family laws, changes in household technologies, changes in earnings inequality and changes in welfare regimes. ${ }^{2}$ Most of this research ignored changes in population supplies over time. Often, they also ignore peer effects in marital behavior.

*We thank Marcin Peski, Robert McCann and seminar participants for useful discussions. We also thank SSHRC for financial support.

${ }^{1}$ Due to the small numbers of cohabitants, the US censuses did not collect data on cohabitation before 1990 .

${ }^{2}$ E.g. Burtless 1999; Choo Siow 2006a; Fernandez, Guner and Knowles 2005; FernandezVillaverde, et. al. 2014; Goldin and Katz 2002; Greenwood, et. al. (2012, 2014); Lundberg and Pollak 2007; Moffitt, et. al. 1998; Stevenson and Wolderers 2007; Waite and Bachrach 2004. 
There were significant changes in population supplies over the time period. The sex ratio (ratio of male to female) of new college graduates have decreased from above one in the seventies to below one currently. See Figure 2 in Appendix $\mathrm{C}$ for women and men between ages 26-30 and 28-32 respectively. This change in the sex ratio may have exacerbated the decline in the marriage rate and also potentially changed marriage matching patterns.

Peer effects, as well as changes in cultural norms, may have also affected cohabitation and other marital behavior (E.g. Waite, et. al. 2000; FernandezVillaverde, et. al. (2014)). Using individual level data, Adamopoulou (2012) shows that there are positive peer effects on the marital decisions of young adults. Also see Drewianka (2003).

The objective of this paper is to first provide an elementary marriage matching function (MMF), the Cobb Douglas MMF, which can be used to parameterize different causes of changes in marital behavior while allowing for peer effects and changes in population supplies. Then we estimate the changes in the parameters of the MMF which capture the evolution of marital behavior in the US between 1990 and 2010 .

Our empirical framework builds on recent research on static behavioral MMFs. Consider a marriage market $s$ at time $t$. There are $I, i=1, . ., I$, types of men and $J, j=1, \ldots, J$, types of women. Let $M^{s t}$ be the population vector of men where a typical element is $m_{i}$, the supply of type $i$ men. $F^{s t}$ is the population vector of women where a typical element is $f_{j}$, the supply of type $j$ women. Each individual can choose to enter a relationship, marriage or cohabitation, $r=[\mathcal{M}, \mathcal{C}]$, and a partner (by type) of the opposite sex for the relationship or not. An unmatched individual chooses a partner of type 0 .

Let $\theta^{s t}$ be a vector of parameters. A marriage matching function (MMF) is a $R_{+}^{2 I J}$ vector valued function $\mu\left(M^{s t}, F^{s t}, \theta^{s t}\right)$ whose typical element is $\mu_{i j}^{r s t}$, the number of $(r, i, j)$ relationships. $\mu_{0 j}^{s t}$ and $\mu_{i 0}^{s t}$ are the numbers of unmatched women and men respectively. $\mu_{i j}^{r s t}$ have to satisfy the following $I+J$ accounting identities:

$$
\begin{aligned}
& \sum_{j=1}^{J} \mu_{i j}^{\mathcal{M} s t}+\sum_{j=1}^{J} \mu_{i j}^{\mathcal{C} s t}+\mu_{i 0}^{s t}=m_{i}^{s t}, 1 \leq i \leq I \\
& \sum_{i=1}^{I} \mu_{i j}^{\mathcal{M} s t}+\sum_{i=1}^{I} \mu_{i j}^{\mathcal{C} s t}+\mu_{0 j}^{s t}=f_{j}^{s t}, \quad 1 \leq j \leq J \\
& \mu_{0 j}^{s t}, \mu_{i 0}^{s t} \geq 0, \quad 1 \leq j \leq J, 1 \leq i \leq I .
\end{aligned}
$$

This paper proposes the Cobb Douglas MMF:

$$
\ln \mu_{i j}^{r s t}=\gamma_{i j}^{r s t}+\alpha_{i j}^{r} \ln \mu_{i 0}^{s t}+\beta_{i j}^{r} \ln \mu_{0 j}^{s t} ; \alpha_{i j}^{r}, \beta_{i j}^{r} \geq 0 \forall(r, i, j)
$$

The Cobb Douglas MMF has some useful properties:

- It nests a large class of behavioral MMFs which makes the parameters easy to interpret. 
- We may interpret $\gamma_{i j}^{r}$ as proportional to the mean gross gains to the couple in relationship $r$ minus the sum of the mean gains to them remaining unmatched for two randomly chosen $(i, j)$ individuals. So one can parametrize $\gamma_{i j}^{r s t}$ to study how a particular behavioral mechanism affects marital matching.

- $\alpha_{i j}^{r}$ and $\beta_{i j}^{r}$ are parameters which are affected by peer and scale effects.

- The numbers of unmatched men and women of each type, $\mu_{0 j}^{s t}$ and $\mu_{i 0}^{s t}$, summarize general equilibrium effects as well as the influence of population supplies.

- The MMF fit any observed marital behavior in a single marriage market. In fact, the model must be restricted to obtain identification even with multi-market data. Luckily, identification is transparent.

- Estimation is also easy. The parameters of the MMF can be estimated using multi-market data by difference in differences and using population supplies as instruments for the unmatched (Section (3)).

- Given population supplies and parameters, the equilibrium marriage matching distribution $\mu\left(M^{s t}, F^{s t}, \theta^{s t}\right)$ exists and is unique. ${ }^{3}$ Although there are $2 \times I \times J$ elements in $\mu$, the analyst only has to first solve a sub-system of $I+J$ non-linear equations whose solution is unique (Lemma 1). The rest of the system is linear. Using this two steps approach, the MMF is easy to simulate for policy evaluations. ${ }^{4}$

While equation (3) is in the Cobb Douglas form, it is not a standard production function. ${ }^{5}$ Rather, equation (3) is a set of equilibrium relationships which define the Cobb Douglas MMF. Therefore it is useful for us to provide some background on this MMF.

The Cobb Douglas MMF started with Choo Siow (2006a, 2006b; hereafter CS). Building on Becker (1973, 1974; reprinted in 1991), CS proposed a frictionless transferable utility MMF. They used McFadden (1972) additive random utility model to model an individual's utility from any type of partner in relationship $r$. For any individual, the idiosyncratic payoff from a partner depends on their type but not their specific identity. The utility a man $g$ of type $i$ will get from relationship $r$ with a woman of type $j, j=0, . ., J$, is:

$$
U_{i j g}^{r}=\tilde{u}_{i j}^{r}-\tau_{i j}^{r}+\epsilon_{i j g}^{r}
$$

\footnotetext{
${ }^{3}$ Uniqueness of equilibrium is not a general property of static frictionless transferable utility models of the marriage market (E.g. Shi and Shum 2014).

${ }^{4}$ Feedback from users of the CS MMF (a special case) suggest that a one step numerical solution is difficult to achieve.

${ }^{5}$ The standard Cobb Douglas model, $\ln \mu_{i j}^{r s t}=\alpha_{i j}^{r} \ln m_{i}^{s t}+\beta_{i j}^{r} \ln f_{j}^{s t}+\gamma_{i j}^{r s t}$, is not a well behaved MMF. In general, it will not satisfy the accounting relationships (1) and (2). Nor does it have spillover effects. I.e. an increase in type $i^{\prime}$ men who are close substitutes for type $i$ men does not affect $\mu_{i j}^{r s t}$.
} 
$\tilde{u}_{i j}^{r}$ is the systematic gross return to a male of type $i$ matched with a female of type $j$ in relationship $r . \tau_{i j}^{r}$ is the transfer made by the man to his partner of type $j$. Let a man who remains unmatched marry a type 0 spouse and $\tau_{i 0}=0$. $\epsilon_{i j g}^{r}$ is an i.i.d. random variable distributed according to the Type 1 extreme value distribution.

Utility from a choice consists of two additive terms, a systematic net component common to all $(r, i, j)$ matches, $u_{i j}^{r}=\tilde{u}_{i j}^{r}-\tau_{i j}^{r}$, and an idiosyncratic component, $\epsilon_{i j g}^{r}$, which is specific to him.

The man $g$ chooses the partner type and relationship which maximizes his utility.

Similarly, the utility which a woman $k$ of type $j$ who chooses to match with a type $i$ man, $i=0, . ., I$, in relationship $r$, is:

$$
V_{i j k}^{r}=\widetilde{v}_{i j}^{r}+\tau_{i j}^{r}+\epsilon_{i j k}^{r}, \text { where }
$$

$\widetilde{v}_{i j}^{r}$ is the systematic gross return to a female of type $j$ matched with a male of type $i$ in relationship $r . \tau_{i j}^{r}$ is the transfer made by her partner to her. A woman who remains unmatched marries a type 0 spouse and $\tau_{0 j}=0$. $\epsilon_{i j k}^{r}$ is an i.i.d. random variable distributed according to the Type 1 extreme value distribution. So in addition to differences in gross systematic gains from marriage, female partners receive transfers, positive or negative, from their male partners.

The marriage market clears when faced with equilibrium transfers, all type $i$ men who want to marry type $j$ women in relationship $r$ will find a woman to match with and vice versa for all $(r, i, j)$.

CS shows that market equilibrium will generate the following MMF:

$$
\begin{aligned}
\ln \frac{\mu_{i j}^{r}}{\sqrt{\mu_{i 0} \mu_{0 j}}} & =\pi_{i j}^{r} \forall(r, i, j) \\
\pi_{i j}^{r} & =\widetilde{u}_{i j}^{r}+\widetilde{v}_{i j}^{r}-\widetilde{u}_{i 0}-\widetilde{v}_{0 j}
\end{aligned}
$$

where $\mu_{i j}^{r}$ is the number of type $i$ men matched to type $j$ women. $\mu_{i 0}$ and $\mu_{0 j}$ are the numbers of unmarried type $i$ men and type $j$ women respectively. $\pi_{i j}^{r}$ are the gross systematic payoffs to marriage for $(i, j)$ couples relative to them remaining unmarried. CS calls $\pi_{i j}^{r}$ the gains to marriage. $\widetilde{u}_{i j}^{r}+\widetilde{v}_{i j}^{r}$ is known as marital output or surplus. As observed by CS, if we only observe the left hand side of (6), only $\pi_{i j}^{r}$, and not its separate components, are identified.

The CS MMF fits any observed marriage distribution in a single marriage market. It obeys constant returns to scale in population vectors.

Assuming no cohabitation and dropping the $r$ superscript, let $\theta$ be the set of $\pi_{i j}$. Decker, et. al. (2013) showed that for any admissible $(M, F, \theta), \mu$ exists and is unique. Decker, et. al. and then Graham (2011) also proceeded to provide comparative statics for the CS model. CS and Siow (forthcoming) show which parameters and characteristics of the marital surplus function, $\widetilde{u}_{i j}+\widetilde{v}_{i j}$, are identified. 
Also ignoring cohabitation, the subsequent theoretical literature has branched in two directions. One branch relaxes the specification of the idiosyncratic component of utility from a partner type choice, $\epsilon_{i j g}$. This branch retains the systematic gross gains from marriage, $\widetilde{u}_{i j}$ and $\widetilde{v}_{i j}$, from CS and the use of transfers to clear the marriage market.

Chiappori, Salanié and Weiss (2012; hereafter CSW) and Graham (2011) extends CS to incorporate heteroskedastic idiosyncratic payoffs by gender and type. Let $\sigma_{i}$ and $\Sigma_{j}$ be the standard deviation of the idiosyncratic payoff for a type $i$ male and type $j$ female respectively. The CSW MMF is:

$$
\ln \mu_{i j}=\frac{\sigma_{i}}{\sigma_{i}+\Sigma_{j}} \ln \mu_{i 0}+\frac{\Sigma_{j}}{\sigma_{i}+\Sigma_{j}} \ln \mu_{0 j}+\frac{\pi_{i j}}{\sigma_{i}+\Sigma_{j}} ; \quad \forall(i, j)
$$

CSW obeys constant returns to scale.

Galichon and Salanié (2013) significantly relaxes the iid Type 1 extreme value distribution of CS and their general model is not nested here. They also show that their MMFs can be derived from a utilitarian social planner choosing marital matches in order to maximize aggregate marital output. In personal communication, Galichon said that all the MMFs in Galichon and Salanié also obey constant returns to scale. In another important extension, Dupuy and Galichon (2012) extends the CS model to account for continuous types.

The second branch studies other behavioral specifications for the systematic net return from relationships, $u_{i j}^{r}=\widetilde{u}_{i j}-\tau_{i j}^{r}$ and $v_{i j}^{r}=\widetilde{v}_{i j}^{r}+\tau_{i j}^{r}$. Again ignoring cohabitation, Dagsvik (2000) assumed that transfers are not available to clear the marriage market. So $\tau_{i j}=0$, and $u_{i j}=\widetilde{u}_{i j}$ and $v_{i j}=\widetilde{v}_{i j}$. He also assumed that the idiosyncratic payoff of man $g$ of type $i$ marrying woman $k$ of type $j$, $\epsilon_{i j g k}$, is distributed i.i.d. Type 1 extreme value. He used the deferred acceptance algorithm to solve for a marriage matching equilibrium and obtained the following non-transferable utility MMF for large marriage markets:

$$
\ln \mu_{i j}=\ln \mu_{i 0}+\ln \mu_{0 j}+\pi_{i j} \forall(i, j)
$$

Menzel (2014) showed that (9) obtains under less restrictive assumptions about the distribution of $\epsilon_{i j g k}$. Call (9) the DM MMF. The DM MMF also fits any observed marriage distribution. Based on simulations, it obeys increasing return to scale in population vectors.

Chiappori and Salanié (2014) has a state of the art survey of the above and related models.

This paper follows the second branch by studying alternative specifications to the systematic net return from relationships. We retain the Type 1 extreme value distribution assumption of CS for the idiosyncratic component of utility from partner choice. Instead, we focus on a different behavioral specification of net utilities, $u_{i j}^{r}$ and $v_{i j}^{r}$. We propose a CS marriage matching model with peer effects (CSPE). Our specification for net utility of a $(r, i, j)$ relationship for a man and a woman is $u_{i j}^{r}=\tilde{u}_{i j}^{r}+\phi_{i}^{r} \ln \mu_{i j}^{r}-\tau_{i j}^{r}$ and $v_{i j}^{r}=\tilde{v}_{i j}^{r}+\Phi_{j}^{r} \ln \mu_{i j}^{r}+\tau_{i j}^{r}$ respectively. We assume that $0 \leq \phi_{i}^{r}, \Phi_{j}^{r} \leq 1$. In this setup, the systematic net 
utilities are affected by how many other individuals of the same type make the same decision.

Our model of multinomial choice with peer effects builds upon Brock and Durlauf (2001). What is new is our application to two sided frictionless matching with equilibrium transfers. The CSPE MMF is:

$$
\ln \mu_{i j}^{r}=\frac{1-\phi_{i}^{0}}{2-\phi_{i}^{r}-\Phi_{j}^{r}} \ln \mu_{i 0}+\frac{1-\Phi_{j}^{0}}{2-\phi_{i}^{r}-\Phi_{j}^{r}} \ln \mu_{0 j}+\frac{\pi_{i j}^{r}}{2-\phi_{i}^{r}-\Phi_{j}^{r}} \forall(r, i, j)
$$

CS, DM and CSW are all special cases of CSPE. Unlike CS and CSW, CSPE does not impose constant returns to scale to population vectors. Due to the presence of peer effects, CSPE is not in the Galichon Salanié class.

When we extend the CS, CSW and DM MMF to additional types of relationships, the log odds of the numbers of different types of relationships, $\ln \left(\mu_{i j}^{\mathcal{M}} / \mu_{i j}^{\mathcal{C}}\right)$, is independent of the sex ratio, $\ln \left(m_{i} / f_{j}\right)$. Independence is a very strong assumption. Arciadiacono, et. al. (2010) shows that independence does not hold for sexual versus non-sexual boy girl relationships in high schools. This paper shows that independence also does not hold for cohabitation versus marriage. CSPE relaxes independence albeit in a restricted manner. The Cobb Douglas MMF relaxes independence more flexibly.

CSPE is a special and testable case of the Cobb Douglas MMF.

When $i$ and $j$ are unidimensional and ordered, supermodularity of the marriage distribution $\mu_{i j}^{r}$, i.e. totally positive of order 2 or $T P 2$, is proportional to the degree of supermodularity of the marital output function, $\widetilde{u}_{i j}^{r}+\widetilde{v}_{i j}^{r}$. Thus supermodularity of the marital output function is testable even in the presence of peer effects in marriage matching.

Mourifié and Siow (in process; CSFT) studies a CS model with piecewise linear frictional transfers. ${ }^{6}$ CSFT is a special case of the Cobb Douglas MMF but not CSPE. So we will not discuss CSFT further here.

The Cobb Douglas MMF nests CS, CSW, DM, CSPE and CSFT as special cases. Since the special cases include frictionless transferable utility models, non-transferable utility models and models with frictional transfers, we should be modest in our ability to determine the importance of transfers in equilibrating the marriage market. Although we are partial to CSPE and it is not rejected empirically, it should be clear that we propose the Cobb Douglas MMF precisely because we do not want to insist on a particular behavioral model of the marriage market.

This paper also makes a methodological contribution. The variational techniques used by Decker, et. al. and Galichon Salanié cannot be directly applied to show existence and uniqueness of equilibrium. Using an alternative approach, we show that the equilibrium marriage distribution of the Cobb Douglas MMF exists and is unique. Building on Graham (2013), we also derive comparative static results for CSPE.

\footnotetext{
${ }^{6}$ Galichon, et. al. (in process) also studies a CS style model with a general frictional transfer technology. They focus on different issues and use a different analytic approach. The two papers are complementary.
} 
We estimate the Cobb Douglas MMF with marriage and cohabitation data across states for women and men between ages 26-30 and 28-32 respectively from the US Censuses in 1990 and 2000, and the American Community Surveys around 2010. Men and women are differentiated by their educational attainment. This empirical analysis builds on Siow and CSW.

Our empirical results show that:

- From a descriptive (goodness of fit) point of view, the Cobb Douglas MMF provides a reasonably complete and parsimonious description of the US marriage market by state from 1990 to 2010 .

- CS, CSW and DM are rejected by the data.

- CSPE is not rejected by the data.

- There are peer and scale effects in US marriage markets.

- Independence of the log odds of the number of cohabitations to the number of marriages to the sex ratio is rejected.

- Consistent with CSW and Siow, to a first order, there is no general increase in positive assortative matching (PAM) by educational attainment from 1990 to 2010.

- Consistent with CSW and many other observers, gains to marriage declined from 1990 to 2010. We further show that gains to cohabitation increased.

The organization of the paper is as follows. The next section presents CSPE. The Cobb Douglas MMF is presented next followed by the empirical application.

\section{Marriage matching with peer effects}

There are $I$ types of men and $J$ types of women. For sake of simplicity we assume only two types of relationship, cohabitation and marriage, $r=[\mathcal{M}, \mathcal{C}]$. Therefore, every individual can decide to cohabit, marry or remain unmatched. For a type $i$ man to match with a type $j$ woman in relationship $r$, he must transfer to her a part of his utility that he values as $\tau_{i j}^{r}$. The woman values the transfer as $\tau_{i j}^{r}$. $\tau_{i j}^{r}$ may be positive or negative.

There are $2 \times I \times J$ matching sub-markets for every combination of relationship, and types of men and women. A matching market clears when, given equilibrium transfers $\tau_{i j}^{r}$, the demand by men of type $i$ for type $j$ women in the relationship $r$ is equal to the supply of type $j$ women for type $i$ men in the relationship $r$ for all $(r, i, j)$. To implement the above framework empirically, we adopt the extreme value random utility model of McFadden to generate market demands for matching partners. Each individual considers matching with a 
member of the opposite gender. Let the utility of male $g$ of type $i$ who matches a female of type $j$ in a relationship $r$ be:

$$
U_{i j g}^{r}=\tilde{u}_{i j}^{r}+\phi_{i}^{r} \ln \mu_{i j}^{r}-\tau_{i j}^{r}+\epsilon_{i j g}^{r}, \text { where }
$$

$\tilde{u}_{i j}^{r}+\phi_{i}^{r} \ln \mu_{i j}^{r}$ : Systematic gross return to a male of type $i$ matching to a female of type $j$ in relationship $r$.

$\phi_{i}^{r}$ : Coefficient of peer effect for relationship $r .1 \geq \phi_{i}^{r} \geq 0$.

$\mu_{i j}^{r}$ : Equilibrium number of $(r, i, j)$ relationships.

$\tau_{i j}^{r}$ : Equilibrium transfer made by a male of type $i$ to a female of type $j$ in relationship $r$.

$\epsilon_{i j g}^{r}$ : i.i.d. random variable distributed according to the Type 1 extreme value distribution.

Due to the peer effect, the net systematic return is increased when more type $i$ men are in the same relationships. It is reduced when the equilibrium transfer $\tau_{i j}^{r}$ is increased.

The above empirical model for multinomial choice with peer effects is standard. See Brock and Durlauf (2001).

And $\widetilde{u}_{i 0}+\phi_{i}^{0} \ln \mu_{i 0}^{0}$ is the systematic payoff that type $i$ men get from remaining unmatched. We allow the peer effect to differ by relationship. For example, unmarried individuals spend more time with their unmarried friends than married individuals with their married friends. On the other hand, due to their higher shadow cost of time, married individuals may want to live in communites with couples like themselves so that local firms will provide services to them. So there is no apriori reason to rank $\phi_{i}^{0}$ versus $\phi_{i}^{r}$.

Individual $g$ will choose according to:

$$
U_{i g}=\max _{j, r}\left\{U_{i 0 g}, U_{i 1 g}^{\mathcal{M}}, \ldots, U_{i j g}^{\mathcal{M}}, \ldots, U_{i J g}^{\mathcal{M}}, U_{i 0 g}^{\mathcal{C}}, \ldots, U_{i j g}^{\mathcal{C}}, \ldots, U_{i J g}^{\mathcal{C}}\right\}
$$

Let $\left(\mu_{i j}^{r}\right)^{d}$ be the number of $(r, i, j)$ matches demanded by $i$ type men and $\left(\mu_{i 0}\right)^{d}$ be the number of unmatched $i$ type men. Following the well known McFadden result, we have:

$$
\begin{aligned}
\frac{\left(\mu_{i j}^{r}\right)^{d}}{m_{i}} & =\mathbb{P}\left(U_{i j g}^{r}-U_{i k g}^{r^{\prime}} \geq 0, k=1, \ldots, J ; r^{\prime}=(\mathcal{M}, \mathcal{C})\right) \\
& =\frac{e^{\tilde{u}_{i j}^{r}+\phi_{i}^{r} \ln \mu_{i j}^{r}-\tau_{i j}^{r}}}{e^{\tilde{u}_{i 0}+\phi_{i}^{0} \ln \mu_{i 0}}+\sum_{r^{\prime} \in\{\mathcal{M}, \mathcal{C}\}} \sum_{k=1}^{J} e^{\tilde{u}_{i k}^{r^{\prime}}+\phi_{i}^{r} \ln \mu_{i j}^{r^{\prime}}-\tau_{i k}^{r^{\prime}}}},
\end{aligned}
$$

where $m_{i}$ denotes the number of men of type $i$. Using (11) we can easily derive the following relationship:

$$
\ln \frac{\left(\mu_{i j}^{r}\right)^{d}}{\left(\mu_{i 0}\right)^{d}}=\tilde{u}_{i j}^{r}-\tilde{u}_{i 0}+\phi_{i}^{r} \ln \mu_{i j}^{r}-\phi_{i}^{0} \mu_{i 0}-\tau_{i j}^{r},
$$

The above equation is a quasi-demand equation by type $i$ men for $(r, i, j)$ relationships. 
The random utility function for women is similar to that for men except that in matching with a type $i$ men in an $(r, i, j)$ relationship, a type $j$ women receives the transfer, $\tau_{i j}^{r}$. Let $\tilde{v}_{i j}^{r}+\Phi_{j}^{r} \ln \mu_{i j}^{r}$ denotes the systematic gross gain that type $j$ women get from matching type $i$ men in the relationship $r . \Phi_{j}^{r}, 1 \geq \Phi_{j}^{r} \geq 0$, is her peer effect coefficient in relationship $(r, i, j)$. And $\tilde{v}_{0 j}+\Phi_{j}^{0} \ln \mu_{0 j}^{0}$ is the systematic payoff that type $j$ women get from remaining unmatched. Let $\left(\mu_{i j}^{r}\right)^{s}$ be the number of $i, j$ matches offered by $j$ type women for the relationship $r$ and $\left(\mu_{0 j}\right)^{s}$ the number of type $j$ women who want to remain unmatched. The quasi-supply equation of type $j$ women for $(r, i, j)$ relationships is given by:

$$
\ln \frac{\left(\mu_{i j}^{r}\right)^{s}}{\left(\mu_{0 j}\right)^{s}}=\tilde{v}_{i j}^{r}-\tilde{v}_{0 j}+\Phi_{j}^{r} \ln \mu_{i j}^{r}-\Phi_{j}^{0} \ln \mu_{0 j}+\tau_{i j}^{r} .
$$

The matching market clears when, given equilibrium transfers $\tau_{i j}^{r}$, the demand of type $i$ men for $(r, i, j)$ relationships is equal to the supply of type $j$ women for $(r, i, j)$ relationships for all $(r, i, j)$ :

$$
\left(\mu_{i j}^{r}\right)^{d}=\left(\mu_{i j}^{r}\right)^{s}=\mu_{i j}^{r} .
$$

Substituting (14) into equations (12) and (13) we get:

$$
\begin{aligned}
\ln \mu_{i j}^{r} & =\frac{1-\phi_{i}^{0}}{2-\phi_{i}^{r}-\Phi_{j}^{r}} \ln \mu_{i 0}+\frac{1-\Phi_{j}^{0}}{2-\phi_{i}^{r}-\Phi_{j}^{r}} \ln \mu_{0 j}+\frac{\pi_{i j}^{r}}{2-\phi_{i}^{r}-\Phi_{j}^{r}} \\
\pi_{i j}^{r} & =\tilde{u}_{i j}^{r}-\tilde{u}_{i 0}+\tilde{v}_{i j}^{r}-\tilde{v}_{0 j}
\end{aligned}
$$

The above is the CS model with peer effects, the CSPE MMF.

When there is no peer effect or all the peer effect coefficients are the same (homogeneous peer effects),

$$
\phi_{i}^{0}=\Phi_{j}^{0}=\phi_{i}^{r}=\Phi_{j}^{r}
$$

we recover the CS MMF. That is,

Proposition 1 No peer effect, or homogenous peer effects, generates observationally equivalent MMFs.

Put another way, the above proposition says if we cannot reject CS using marriage matching data alone, we also cannot reject homogenous peer effects. This lack of identification is our version of the reflection problem in Manski's linear-in-mean peer effects model. See Manski (1993).

On the other hand,

Corollary 1 When $\frac{1-\phi_{i}^{0}}{2-\phi_{i}^{r}-\Phi_{j}^{r}} \neq \frac{1}{2}$ and/or $\frac{1-\Phi_{j}^{0}}{2-\phi_{i}^{r}-\Phi_{j}^{r}} \neq \frac{1}{2}$, non-homogenous peer effects are present. 
The above corollary says that non-homogenous peer effects are generically detectable. This corollary is related to identification of linear models with nonhomogenous peer effects. ${ }^{7}$

When

$$
\frac{1-\phi_{i}^{0}}{2-\phi_{i}^{r}-\Phi_{j}^{r}}=\frac{1-\Phi_{j}^{0}}{2-\phi_{i}^{r}-\Phi_{j}^{r}}=1
$$

we recover the DM MMF. Intuitively in this case, we want the peer effect on relationships to be significantly more powerful than that for remaining unmatched. E.g. $\phi_{i}^{0}=\Phi_{j}^{0}=0$ and $\phi_{i}^{r}=\Phi_{j}^{r}=\frac{1}{2}$.

Also, when

$$
\phi_{i}^{0}+\Phi_{j}^{0}=\phi_{i}^{r}+\Phi_{j}^{r}=\phi_{i}^{r^{\prime}}+\Phi_{j}^{r^{\prime}},
$$

CSW MMF obtains.

From (15), you cannot distinguish $\phi_{i}^{r}$ from $\Phi_{j}^{r}$. On the other hand, you can test whether $\phi_{i}^{0}=\Phi_{j}^{0}$.

And from (15),

$$
\begin{aligned}
\ln \frac{\mu_{i j}^{\mathcal{M}}}{\mu_{i j}^{\mathcal{C}}} & =\frac{\left(\phi_{i}^{\mathcal{M}}+\Phi_{j}^{\mathcal{M}}-\phi_{i}^{\mathcal{C}}-\Phi_{j}^{\mathcal{C}}\right)}{\left(2-\phi_{i}^{\mathcal{M}}-\Phi_{j}^{\mathcal{M}}\right)\left(2-\phi_{i}^{\mathcal{C}}-\Phi_{j}^{\mathcal{C}}\right)}\left[\left(1-\phi_{i}^{0}\right) \ln \mu_{i 0}+\left(1-\Phi_{j}^{0}\right) \ln \mu_{0 j}\right] \\
& +\frac{\pi_{i j}^{\mathcal{M}}}{2-\phi_{i}^{\mathcal{M}}-\Phi_{j}^{\mathcal{M}}}-\frac{\pi_{i j}^{\mathcal{C}}}{2-\phi_{i}^{\mathcal{C}}-\Phi_{j}^{\mathcal{C}}}
\end{aligned}
$$

Since $\mu_{i 0}$ and $\mu_{0 j}$ appears on the right hand side of (17), the log odds of the number of $r$ to $r^{\prime}$ relationships will not be independent of the sex ratio.

It is easy to check that under CS, CSW and DM, the log odds of the number of $r$ to $r^{\prime}$ relationships is independent of the sex ratio. Independence is a very strong assumption and unlikely to hold every two types of relationships. CSPE relaxes the independence assumption. However because the coefficients on unmatched men and women have the same sign, this independence is restricted.

We will now study PAM patterns. Let the heterogeneity across males (females) be one dimensional and ordered. Without loss of generality, let male (female) ability be increasing in $i(j)$.

Also let:

$$
\phi_{i}^{0}=\phi^{0} ; \Phi_{j}^{0}=\Phi^{0} ; \phi_{i}^{r}=\phi^{r} ; \Phi_{j}^{r}=\Phi^{r}
$$

that is, the peer effects depend on their gender and the relationship they pick but not their type or their partner's type. We call equation (18) type independent peer effects. Type independence peer effects leads to type independent exponents in the Cobb Douglas MMF, a testable restriction.

\footnotetext{
${ }^{7}$ Blume, et. al. (forthcoming) has a state of the art survey. Also see Djebbari, et. al. (2009).
} 
Then using (15), the local log odds for $(r, i, j)$ is:

$$
\begin{aligned}
l(r, i, j) & =\ln \frac{\mu_{i j}^{r} \mu_{i+1, j+1}^{r}}{\mu_{i+1, j}^{r} \mu_{i, j+1}^{r}}=\frac{\pi_{i j}^{r}+\pi_{i+1, j+1}^{r}-\pi_{i+1, j}^{r}-\pi_{i, j+1}^{r}}{2-\phi^{r}-\Phi^{r}} \\
& =\frac{\tilde{u}_{i j}^{r}+\tilde{v}_{i j}^{r}+\tilde{u}_{i+1, j+1}^{r}+\tilde{v}_{i+1, j+1}^{r}-\left(\tilde{u}_{i+1, j}^{r}+\tilde{v}_{i+1, j}^{r}\right)-\left(\tilde{u}_{i, j+1}^{r}+\tilde{v}_{i, j+1}^{r}\right)}{2-\phi^{r}-\Phi^{r}}
\end{aligned}
$$

According to (19), if the marital output function, $\tilde{u}_{i j}^{r}+\tilde{v}_{i j}^{r}$, is supermodular in $i$ and $j$, then the local log odds, $l(r, i, j)$, are positive for all $(i, j)$, or totally positive of order 2 ( $T P 2)$. Statisticians use $T P 2$ as a measure of stochastic positive assortative matching. Thus even when peer effects are present, we can test for supermodularity of the marital output function, a cornerstone of Becker's theory of positive assortative matching in marriage. This result generalizes Siow (forthcoming), CSW and Graham (2011).

CSPE MMF is a special case of the Cobb Douglas MMF. We will show the existence and uniqueness of the marriage matching distribution of the Cobb Douglas MMF and derive some comparative statics results.

\section{The Cobb Douglas MMF}

Consider the Cobb Douglas MMF defined by:

$$
\begin{gathered}
\ln \frac{\mu_{i j}^{r}}{\left(\mu_{i 0}\right)^{\alpha_{i j}^{r}}\left(\mu_{0 j}\right)^{\beta_{i j}^{r}}}=\gamma_{i j}^{r} \forall(r, i, j) \\
\alpha_{i j}^{r}, \beta_{i j}^{r} \geq 0
\end{gathered}
$$

Consistent with the behavioral models, and the fact that $\gamma_{i j}^{r}$ can be negative, we interpret $\gamma_{i j}^{r}$ as proportional to the mean gross gains to relationship $r$ minus the sum of the mean gains to them remaining unmatched for two randomly chosen $(i, j)$ individuals.

The matching equilibrium in this model is characterized by the Cobb Douglas MMF (21) and the population constraint equations. Showing the existence of an equilibrium matching and its uniqueness is not an easy task. The existence and uniqueness of the equilibrium matching in the CS model has been well studied by Decker et. al. (2013), and Galichon and Salanié (2012). However, those two approaches cannot be directly applied to show existence and uniqueness of equilibrium in this context. To the best of our knowledge, nothing was known about the existence and the uniqueness of the equilibrium for the Cobb Douglas MMF. We propose an approach that proves the existence and uniqueness of this model. This new approach will also serve as an alternative to the existence and uniqueness proofs proposed by Decker et. al. (2013) and Galichon and Salanié (2012) for the CS MMF. The details of the complete development of our approach are derived in Appendix A. 
Following CS, an important simplification in the proof is to first reduce the $2 r \times I \times J$ system of non-linear equations to an $I+J$ system of the numbers of unmatched individuals by substituting the Cobb Douglas MMF in equation (21) into the population constraints, (1) and (2), to get:

\section{Lemma 1}

$$
\begin{aligned}
& m_{i}=\mu_{i 0}+\sum_{j=1}^{J} \mu_{i 0}^{\alpha_{i j}^{\mathcal{M}}} \mu_{0 j}^{\beta_{i j}^{\mathcal{M}}} e^{\gamma_{i j}^{\mathcal{M}}}+\sum_{j=1}^{J} \mu_{i 0}^{\alpha_{i j}^{\mathcal{C}}} \mu_{0 j}^{\beta_{i j}^{\mathcal{C}}} e^{\gamma_{i j}^{\mathcal{C}}}, \text { for } 1 \leq i \leq I, \\
& f_{j}=\mu_{0 j}+\sum_{i=1}^{I} \mu_{i 0}^{\alpha_{i j}^{\mathcal{M}}} \mu_{0 j}^{\beta_{i j}^{\mathcal{M}}} e^{\gamma_{i j}^{\mathcal{M}}}+\sum_{i=1}^{I} \mu_{i 0}^{\alpha_{i j}^{\mathcal{c}}} \mu_{0 j}^{\beta_{i j}^{\mathcal{C}}} e^{\gamma_{i j}^{\mathcal{c}}}, \text { for } 1 \leq j \leq J .
\end{aligned}
$$

After we solve for the equilibrium numbers of unmatched individuals, $I+J$ of them, the equilibrium numbers of $2 r \times I \times J$ matches can be solved one at a time using (21).

The following theorem summarizes our results:

Theorem 1 [Existence and Uniqueness of the Equilibrium matching] For every fixed matrix of relationship gains and coefficients $\beta_{i j}^{r} ; \alpha_{i j}^{r} \geq 0$, the equilibrium matching of the Cobb Douglas MMF model exists and is unique.

Remark 1 From an application point of view, the importance of the theorem is that we can simulate the model by first solving an $I+J$ system of non-linear equations which has a unique solution rather than an $2 r \times I \times J$ system. The two steps approach to solving for the equilibrium matching is highly recommended. Feedback from users of CS complain about the difficulty of numerically solving the $I \times J$ system without cohabitation in one step.

Notice that using (21),

$$
\ln \frac{\mu_{i j}^{r}}{\mu_{i j}^{r^{\prime}}}=\left(\alpha_{i j}^{r}-\alpha_{i j}^{r^{\prime}}\right) \ln \left(\mu_{i 0}\right)+\left(\beta_{i j}^{r}-\beta_{i j}^{r^{\prime}}\right) \ln \left(\mu_{0 j}\right)+\gamma_{i j}^{r}-\gamma_{i j}^{r^{\prime}} \forall(r, i, j)
$$

So:

Lemma 2 When $\left(\alpha_{i j}^{r}-\alpha_{i j}^{r^{\prime}}\right)=\left(\beta_{i j}^{r}-\beta_{i j}^{r^{\prime}}\right)=0$ as in CS, CSW and DM, the log odd of $\mu_{i j}^{r}$ to $\mu_{i j}^{r^{\prime}}$ is independent of the sex ratio $m_{i} / f_{j}$. Otherwise the log odd is not independent of the sex ratio.

Independence is a very strong assumption and unlikely to hold every two types of relationships. Arciadiacono, et. al. (2010) shows that independence does not hold for sexual versus non-sexual boy girl relationships in high schools. We show here that it does not hold for cohabitation versus marriage. CSPE and CSFT provide two behavioral models which relaxes independence. Indeed, whenever $\left(\alpha_{i j}^{r}-\alpha_{i j}^{r^{\prime}}\right) \neq 0$ or $\left(\beta_{i j}^{r}-\beta_{i j}^{r^{\prime}}\right) \neq 0, m_{i} / f_{j}$ affects $\ln \left(\mu_{i j}^{r} / \mu_{i j}^{r^{\prime}}\right)$ through its impact on the unmatched $\mu_{i 0}$ and $\mu_{0 j}$. 
With multi-market data, $\beta_{i j}^{r}$ and $\alpha_{i j}^{r}$ are identified under some mild restrictions, as shall be clearer soon. However the fully flexible Cobb Douglas MMF cannot be estimated precisely with the data which we have. So often, we will assume that the exponents on the Cobb Douglas MMF are gender and relationship specific but independent of the types of couples, $(i, j): \beta_{i j}^{r}=\beta^{r}$ and $\alpha_{i j}^{r}=\alpha^{r}$.

Type independent exponents is obtained under CSPE when we impose type independent peer effects:

$$
\phi_{i}^{0}=\phi^{0} ; \Phi_{j}^{0}=\Phi^{0} ; \phi_{i}^{r}=\phi^{r} ; \Phi_{j}^{r}=\Phi^{r}
$$

With multimarket data, type independent exponents, $\beta_{i j}^{r}=\beta^{r}$ and $\alpha_{i j}^{r}=$ $\alpha^{r}$, is in principle a testable relationship. From a practical point of view, the most flexible model that we estimate in this paper imposes type independent exponents. $^{8}$

When we impose type independent exponents, the local log odds, $l(r, i, j)$, of the Cobb Douglas MMF become:

$$
l(r, i, j)=\ln \frac{\mu_{i j}^{r} \mu_{i+1, j+1}^{r}}{\mu_{i+1, j}^{r} \mu_{i, j+1}^{r}}=\gamma_{i j}^{r}+\gamma_{i+1, j+1}^{r}-\gamma_{i+1, j}^{r}-\gamma_{i, j+1}^{r}
$$

Following the interpretation of the local log odds for CSPE in equation (19), and all the other behavioral MMFs considered in this paper, we interpret $l(r, i, j)$ as proportional to the degree of local complementarity of the marital output function of the couple at $(r, i, j)$.

Comparing this MMF with the above MMFs, CSPE, CS, DM and CSW are all special cases. It is convenient to summarize the different models and some of their properties.

\begin{tabular}{|l|l|l|l|l|}
\hline \multicolumn{5}{|c|}{ Models and restrictions on $\alpha^{r}$ and $\beta^{r}$} \\
\hline Model & $\alpha^{r}$ & $\beta^{r}$ & $\gamma_{i j}^{r}$ & Restrictions \\
\hline Cobb Douglas MMF & $\alpha^{r}$ & $\beta^{r}$ & $\gamma_{i j}^{r}$ & $\alpha^{r} \geq 0, \beta^{r} \geq 0$ \\
\hline CS & $\frac{1}{2}$ & $\frac{1}{2}$ & $\pi_{i j}^{r}$ & $\alpha^{r}=\beta^{r}=\frac{1}{2}$ \\
\hline DM & 1 & 1 & $\pi_{i j}^{r}$ & $\alpha^{r}=\beta^{r}=1$ \\
\hline CSW & $\frac{\sigma}{\sigma+\Sigma}$ & $\frac{\Sigma}{\sigma+\Sigma}$ & $\frac{\pi_{i j}}{\sigma+\Sigma}$ & $\alpha, \beta>0 ; \alpha+\beta=1$ \\
\hline CSPE & $\frac{1-\phi^{0}}{2-\phi^{r}-\Phi}$ & $\frac{1-\Phi^{0}}{2-\phi^{r}-\Phi^{r}}$ & $\frac{\pi_{i j}^{r}}{2-\phi^{r}-\Phi^{r}}$ & $\alpha^{r}, \beta^{r} \geq 0, \frac{\alpha^{\mathcal{M}}}{\alpha^{c}}=\frac{\beta^{\mathcal{M}}}{\beta^{C}}$ \\
\hline
\end{tabular}

In particular, from (15), CSPE implies:

$$
\frac{\alpha^{r}}{\alpha^{r^{\prime}}}=\frac{\beta^{r}}{\beta^{r^{\prime}}}
$$

which is a testable restriction.

Since different cases of the Cobb Douglas MMF imply the presence of scale effects or otherwise, we provide a restriction for scale effects. Then:

\footnotetext{
${ }^{8}$ We estimated the fully flexible CSPE model but the point estimates on the unmatched interacted with the type of the match were too imprecisely estimated to be useful.
} 
Proposition 2 (Constant return to scale) The equilibrium matching distribution of the Cobb Douglas MMF model satisfies the Constant return to scale property if $\beta^{r}+\alpha^{r}=1$ i.e.

$$
\beta^{r}+\alpha^{r}=1 \text { for } r \in\{\mathcal{M}, \mathcal{C}\} \Rightarrow \sum_{i=1}^{I} \frac{\partial \mu}{\partial m_{i}} m_{i}+\sum_{j=1}^{J} \frac{\partial \mu}{\partial f_{j}} f_{j}=\mu .
$$

The result claims that the Cobb Douglas MMF model exhibits constant results to scale if $\beta^{r}+\alpha^{r}=1$, meaning that, holding the type distributions of men and women fixed, increasing market size has no effect on the probability of forming relationship $r$. The proposition generalizes to $\beta_{i j}^{r}+\alpha_{i j}^{r}=1$ for all $(r, i, j)$ implies constant returns to scale.

Remark 2 A caution on testing for increasing or decreasing returns to scale: Unlike standard Cobb Douglas, so far we have been unable to show that $\beta^{r}+$ $\alpha^{r}>1$ and $\beta^{r}+\alpha^{r}<1$ implies increasing and decreasing returns to scale respectively. ${ }^{9}$

We can provide other comparative statics results for the Cobb Douglas MMF model. To derive the different comparative statics, we generalize the Graham (2013) approach and then show the following results:

Theorem 2 Let $\mu$ be the equilibrium matching distribution of the Cobb Douglas $M M F$ model. If the coefficients $\beta^{r}$ and $\alpha^{r}$ respect the restrictions

1. $0<\beta^{r} ; \alpha^{r} \leq 1$ for $r \in\{\mathcal{M}, \mathcal{C}\}$;

2. $\max \left(\beta^{\mathcal{C}}-\alpha^{\mathcal{C}}, \beta^{\mathcal{M}}-\alpha^{\mathcal{M}}\right)<\min _{i \in I}\left(\frac{1-\rho_{i}^{m}}{\rho_{i}^{m}}\right)$;

3. $\min \left(\beta^{\mathcal{C}}-\alpha^{\mathcal{C}}, \beta^{\mathcal{M}}-\alpha^{\mathcal{M}}\right)>-\max _{j \in J}\left(\frac{1-\rho_{j}^{f}}{\rho_{j}^{f}}\right)$;

where $\rho_{i}^{m}$ is the rate of matched men of type $i$ and $\rho_{j}^{f}$ is the rate of matched women of type $j$, then the following inequalities hold in the neighbourhood of $\mu^{e q}$ :

1. Type-specific elasticities of unmatched.

$$
\begin{aligned}
& \text { (a) } \frac{m_{i}}{\mu_{k 0}} \frac{\partial \mu_{k 0}}{\partial m_{i}} \geq \begin{cases}\frac{1}{m_{i}^{*}} \frac{m_{k}}{m_{k}^{*}} \sum_{j=1}^{J} \frac{\left[\alpha^{\mathcal{M}} \mu_{k j}^{\mathcal{M}}+\alpha^{\mathcal{C}} \mu_{k j}^{\mathcal{C}}\right]\left[\beta^{\mathcal{M}} \mu_{k j}^{\mathcal{M}}+\beta^{\mathcal{C}} \mu_{k j}^{\mathcal{C}}\right]}{f_{j}^{*}}>0 & \text { if } k \neq i \\
\frac{m_{i}^{*}}{m_{i}^{*}}\left[1+\frac{1}{m_{i}^{*}} \sum_{j=1}^{J} \frac{\left[\alpha^{\mathcal{M}} \mu_{i j}^{\mathcal{M}}+\alpha^{\mathcal{C}} \mu_{i j}^{\mathcal{C}}\right]\left[\beta^{\mathcal{M}} \mu_{i j}^{\mathcal{M}}+\beta^{\mathcal{C}} \mu_{i j}^{\mathcal{C}}\right]}{f_{j}^{*}}\right]>1 & \text { if } k=i,\end{cases} \\
& 1 \leq k \leq I .
\end{aligned}
$$

\footnotetext{
${ }^{9}$ We have investigated two definitions of increasing returns: (1) The unmatched rates for men and women must fall by more than proportional to the increase in population supplies. (2) The unmatched rates for all types of individuals must fall by more than proportional to the increase in population supplies.
} 
(b) $\frac{f_{j}}{\mu_{0 k}} \frac{\partial \mu_{0 k}}{\partial f_{j}} \geq \begin{cases}\frac{1}{f_{j}^{*}} \frac{f_{k}}{f_{k}^{*}} \sum_{i=1}^{I} \frac{\left[\alpha^{\mathcal{M}} \mu_{i k}^{\mathcal{M}}+\alpha^{\mathcal{C}} \mu_{i k}^{\mathcal{C}}\right]\left[\beta^{\mathcal{M}} \mu_{i k}^{\mathcal{M}}+\beta^{\mathcal{C}} \mu_{i k}^{\mathcal{C}}\right]}{m_{i}^{*}}>0 & \text { if } k \neq j \\ \frac{f_{j}}{f_{j}^{*}}\left[1+\frac{1}{f_{j}^{*}} \sum_{i=1}^{I} \frac{\left[\alpha^{\mathcal{M}} \mu_{i j}^{\mathcal{M}}+\alpha^{\mathcal{C}} \mu_{\mu_{j}}^{\mathcal{C}}\right]\left[\beta^{\mathcal{M}} \mu_{i j}^{\mathcal{M}}+\beta^{\mathcal{C}} \mu_{i j}^{\mathcal{C}}\right]}{m_{i}^{*}}\right]>1 & \text { if } k=j,\end{cases}$ $1 \leq k \leq J$

(c)

$$
\frac{m_{i}}{\mu_{0 j}} \frac{\partial \mu_{0 j}}{\partial m_{i}} \leq-\frac{\left[\alpha^{\mathcal{M}} \mu_{i j}^{\mathcal{M}}+\alpha^{\mathcal{C}} \mu_{i j}^{\mathcal{C}}\right]}{m_{i}^{*} f_{j}^{*}} m_{i}<0, \text { for } 1 \leq i \leq I \text { and } 1 \leq j \leq J,
$$

(d)

$$
\frac{f_{j}}{\mu_{i 0}} \frac{\partial \mu_{i 0}}{\partial f_{j}} \leq-\frac{\left[\beta^{\mathcal{M}} \mu_{i j}^{\mathcal{M}}+\beta^{\mathcal{C}} \mu_{i j}^{\mathcal{C}}\right]}{m_{i}^{*} f_{j}^{*}} f_{j}<0, \text { for } 1 \leq i \leq I \text { and } 1 \leq j \leq J,
$$

2. Variation of the $\log$ ratio $\ln \frac{\mu_{i j}^{\mathcal{M}}}{\mu_{i j}^{\mathcal{C}}}$ :

If $\alpha^{\mathcal{M}}>\alpha^{\mathcal{C}}$ and $\beta^{\mathcal{C}}>\beta^{\mathcal{M}}$ we have

(a) $\frac{1}{\partial m_{i}}\left[\ln \frac{\mu_{k j}^{\mathcal{M}}}{\mu_{k j}^{\mathcal{C}}}\right] \geq\left\{\begin{array}{cc}\frac{\alpha^{\mathcal{M}}-\alpha^{\mathcal{C}}}{m_{i}^{*} m_{i}} \frac{m_{k}}{m_{k}^{*}} \sum_{j=1}^{J} \frac{\left[\alpha^{\mathcal{M}} \mu_{k j}^{\mathcal{M}}+\alpha^{\mathcal{C}} \mu_{k j}^{\mathcal{C}}\right]\left[\beta^{\mathcal{M}} \mu_{k j}^{\mathcal{M}}+\beta^{\mathcal{C}} \mu_{k j}^{\mathcal{C}}\right]}{f_{j}^{*}} & \\ +\left(\beta^{\mathcal{M}}-\beta^{\mathcal{C}}\right) \frac{\left[\alpha^{\mathcal{M}} \mu_{i j}^{\mathcal{M}}+\alpha^{\mathcal{C}} \mu_{i j}^{\mathcal{c}}\right]}{m_{i}^{*} f_{j}^{*}}>0 & \text { if } k \neq i \\ \frac{\alpha_{-\alpha}^{\mathcal{M}}-\alpha^{\mathcal{C}}}{m_{i}^{*}}\left[\begin{array}{c}\left.1+\frac{1}{m_{i}^{*}} \sum_{j=1}^{J} \frac{\left.\left.\left[\alpha^{\mathcal{M}} \mu_{i j}^{\mathcal{M}}+\alpha^{\mathcal{C}} \mu_{i j}^{\mathcal{C}}\right]\right] \beta^{\mathcal{M}} \mu_{i j}^{\mathcal{M}}+\beta^{\mathcal{C}} \mu_{i j}^{\mathcal{C}}\right]}{f_{j}^{*}}\right] \\ +\left(\beta^{\mathcal{M}}-\beta^{\mathcal{C}}\right) \frac{\left[\alpha^{\mathcal{M}} \mu_{i j}^{\mathcal{M}}+\alpha^{\mathcal{C}} \mu_{i j}^{\mathcal{c}}\right]}{m_{i}^{*} f_{j}^{*}}>\alpha^{\mathcal{M}}-\alpha^{\mathcal{C}}\end{array} \quad \text { if } k=i,\right.\end{array}\right.$

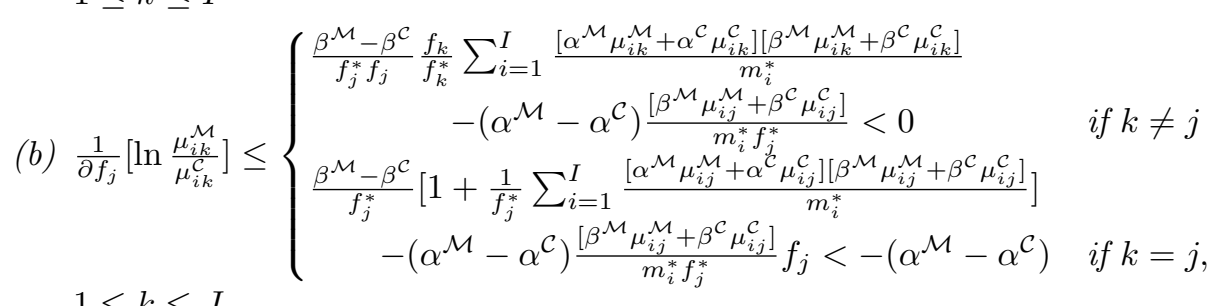

where

$$
\begin{aligned}
m_{i}^{*} & \equiv m_{i}-\sum_{j=1}^{J}\left[\left(1-\alpha^{\mathcal{M}}\right) \mu_{i j}^{\mathcal{M}}+\left(1-\alpha^{\mathcal{C}}\right) \mu_{i j}^{\mathcal{C}}\right], \text { for } 1 \leq i \leq I, \\
f_{j}^{*} & \equiv f_{j}-\sum_{i=1}^{I}\left[\left(1-\beta^{\mathcal{M}}\right) \mu_{i j}^{\mathcal{M}}+\left(1-\beta^{\mathcal{C}}\right) \mu_{i j}^{\mathcal{C}}\right], \text { for } 1 \leq j \leq J .
\end{aligned}
$$

Results 1a and 1b say that the unmatched rate for any type of individual is increasing in the supply of any type of individual of the same gender. Results $1 \mathrm{c}$ 
and $1 \mathrm{~d}$ say that the unmatched rate for any type of individual is decreasing in the supply of any type of individual of the opposite gender. Result 2 provides some comparative statics on the log odds of the number of marriages to cohabitations with respect to changes in population supplies.

The above results generalize Theorem 2 of Decker et. al. (2012), and the case i) of Theorem 1 of Graham (2013). It is worth noting that the restriction imposed on $\beta^{r}$ and $\alpha^{r}$ are only necessary and would be very mild depending on the model. For instance, those restrictions directly holds for the CS and DM model; Graham (2013) shows that those restrictions are not necessary to derive the comparative statistics in the CSW model; for the CSFT model Mourifié and Siow (in process) show that this restriction only avoid to have simultaneously high friction coefficients and marriage rates. This is reasonable since having high level of friction coefficients in the CSFT model decreases matching rates. Moreover, since we will show that the coefficient $\beta^{r}$ and $\alpha^{r}$ could be estimated, those restrictions are therefore testable.

\section{Identification and estimation}

Consider the general Cobb Douglas MMF in presence of multi market data:

$$
\ln \mu_{i j}^{r s t}=\alpha_{i j}^{r} \ln \mu_{i 0}^{s t}+\beta_{i j}^{r} \ln \mu_{0 j}^{s t}+\gamma_{i j}^{r s t} .
$$

As discussed in the introduction, although equation (25) is in the Cobb Douglas form, it is not a production function relationship. Rather, it is a set of equilibrium relationships which defines the MMF. This section provides flexible specifications which are identified and can be estimated using a difference in differences instrumental variables methodology.

Even with multimarket data, the most general Cobb Douglas MMF is not identified. There are $2 \times I \times J \times S \times T$ elements in the observed matching distribution (i.e. $\mu_{i j}^{r s t}$ ) and there are $2 \times I \times J \times S \times T+4 \times I \times J$ parameters i.e. $\left(\gamma_{i j}^{r s t}, \alpha_{i j}^{r}\right.$, and $\left.\beta_{i j}^{r}\right)$. Therefore, to obtain identification of the general Cobb Douglas MMF we will impose additional mild restrictions on the structure of the gains i.e. $\gamma_{i j}^{r s t}$.

\section{Assumption 1 Let:}

1. (Additive separability of the gain). $\gamma_{i j}^{r s t}=\pi_{i j}^{r}+\eta_{i j}^{r s}+\zeta_{i j}^{r t}+\epsilon_{i j}^{r s t}$ where $\pi_{i j}^{r}$ represents the type fixed effect, $\eta_{i j}^{r s}$ the state fixed effect, $\zeta_{i j}^{r t}$ the time fixed effect, and $\epsilon_{i j}^{r s t}$ the residual terms.

2. (Instrumental Variable $(I V)) . \mathbb{E}\left[\epsilon_{i j}^{r s t} \mid z_{i j}^{11}, \ldots, z_{i j}^{S T}\right]=0$, where $z_{i j}^{s t}=\left(m_{i}^{s t}, f_{j}^{s t}\right)^{\prime}$.

Assumption 1 (1) decomposes $\gamma_{i j}^{r s t}$ into match type fixed effect, state fixed effect and time fixed effect, and the error term of the regression, $\epsilon_{i j}^{r s t}$. When $\epsilon_{i j}^{r s t}$ 
increases, the gain to the match increases which will increase $\mu_{i j}^{r s t}$ and therefore likely reduces $\mu_{i 0}^{r s t}$ and $\mu_{0 j}^{r s t}$. Thus $\mu_{i 0}^{r s t}$ and $\mu_{0 j}^{r s t}$ and the error term $\epsilon_{i j}^{r s t}$ are likely negatively correlated. So in general, using OLS to estimate equation (25) is inconsistent.

Assumption 1 (2) allows us to use the population supplies, $m_{i}^{s t}$ and $f_{j}^{s t}$, as instruments for $\mu_{i 0}^{r s t}$ and $\mu_{0 j}^{r s t}$. The assumption says that the population supplies must be orthogonal to $\epsilon_{i j}^{r s t}$.

Under Assumption 1 (1), first let subtract from equation (25) the timeaveraged model $\overline{\ln } \mu_{i j}^{r s}=\alpha_{i j}^{r} \overline{\ln } \mu_{i 0}^{s}+\beta_{i j}^{r} \overline{\ln } \mu_{0 j}^{s}+\pi_{i j}^{r}+\eta_{i j}^{r s}+\bar{\zeta}_{i j}^{r}+\bar{\epsilon}_{i j}^{r s}$. Then,

$$
\ln \mu_{i j}^{r s t}-\overline{\ln } \mu_{i j}^{r s}=\alpha_{i j}^{r}\left[\ln \mu_{i 0}^{s t}-\overline{\ln } \mu_{i 0}^{s}\right]+\beta_{i j}^{r}\left[\ln \mu_{0 j}^{s t}-\overline{\ln } \mu_{0 j}^{s}\right]+\zeta_{i j}^{r t}-\bar{\zeta}_{i j}^{r}+\epsilon_{i j}^{r s t}-\bar{\epsilon}_{i j}^{r s} .
$$

Second, subtract from equation (26) the state-averaged model of equation (26) $\overline{\ln } \mu_{i j}^{r t}-\overline{\overline{\ln }} \mu_{i j}^{r}=\alpha_{i j}^{r}\left[\overline{\ln } \mu_{i 0}^{t}-\overline{\overline{\ln }} \mu_{i 0}\right]+\beta_{i j}^{r}\left[\overline{\ln } \mu_{0 j}^{t}-\overline{\overline{\ln }} \mu_{0 j}\right]+\zeta_{i j}^{r t}-\bar{\zeta}_{i j}^{r}+\bar{\epsilon}_{i j}^{r t}-\overline{\bar{\epsilon}}_{i j}^{r}$ We have then,

$$
\widetilde{y_{i j}^{r s t}}=\widetilde{x_{i j}^{s t}} \widetilde{\lambda_{i j}^{r}}+\widetilde{\epsilon_{i j}^{r s t}}
$$

where $\widetilde{y_{i j}^{r s t}} \equiv \ln \mu_{i j}^{r s t}-\overline{\ln } \mu_{i j}^{r s}-\overline{\ln } \mu_{i j}^{r t}+\overline{\overline{\ln }} \mu_{i 0}, \widetilde{x_{i j}^{s t}} \equiv\left(\ln \mu_{i 0}^{s t}-\overline{\ln } \mu_{i 0}^{s}-\overline{\ln } \mu_{i 0}^{t}+\right.$ $\left.\overline{\overline{\ln }} \mu_{i 0}, \ln \mu_{0 j}^{s t}-\overline{\ln } \mu_{0 j}^{s}-\overline{\ln } \mu_{0 j}^{t}+\overline{\overline{\ln }} \mu_{0 j}\right)^{\prime}, \widetilde{\lambda_{i j}^{r}} \equiv\left(\alpha_{i j}^{r}, \beta_{i j}^{r}\right)^{\prime}$, and $\widetilde{\epsilon_{i j}^{r s t}} \equiv\left[\epsilon_{i j}^{r s t}-\bar{\epsilon}_{i j}^{r s}\right]-$ $\left[\bar{\epsilon}_{i j}^{r t}-\overline{\bar{\epsilon}}_{i j}^{r}\right]$.

Since $\mu_{i 0}^{s t}$ and $\mu_{0 j}^{s t}$ are potentially correlated with the residual terms $\epsilon_{i j}^{r s t}$ a simple ordinary least square (OLS) will not be able to identify $\widetilde{\lambda_{i j}^{r}}$. Therefore, we will instrument $\mu_{i 0}^{s t}$ and $\mu_{0 j}^{s t}$ respectively with $m_{i}^{s t}$ and $f_{j}^{s t}$. Notice that to be a valid instrument $\mu_{i 0}^{s t}$ and $\mu_{0 j}^{s t}$ should be respectively correlated with $\mu_{i 0}^{s t}$ and $\mu_{0 j}^{s t}$ and respect the exogeneity condition summarizes in Assumption 1 (2). As can be seen in Theorem 2, the comparative statics show the correlation between $m_{i}^{s t}$ and $f_{j}^{s t}$ and the unmatched. Therefore, $\widetilde{\lambda_{i j}^{r}}$ can be identify using the IV estimand if $\mathbb{E}\left[z_{i j}^{s t} \widetilde{x_{i j}^{s t}}\right]$ is of full column rank. The identification result is summarized in the following proposition.

Proposition 3 Under Assumption 1, the general Cobb Douglas MMF is identified if $\mathbb{E}\left[z_{i j}^{s t} \widetilde{x_{i j}^{s t}}\right]$ is of full column rank. The identification equation is given by $\widetilde{\lambda_{i j}^{r}}=\left\{\mathbb{E}\left[z_{i j}^{s t} \widetilde{x_{i j}^{s t}}\right]\right\}^{-1} \mathbb{E}\left[z_{i j}^{s t} \widetilde{y_{i j}^{r s t}}\right]$.

We have a few comments. First, whenever $\widetilde{\lambda_{i j}^{r}}$ is identified, we can identify the gain matrix $\gamma_{i j}^{r s t}$ using equation (25). Second, this model can also be estimated using the generalized method of moments (GMM). Third, whenever the numbers of state $S$ and period $T$ are not high, we do not need to do the double differentiation. We can use a sequence of state and time dummies fixed-effects.

\section{Empirical results}

We study the marriage matching behavior of 26-30 years old women and 28-32 years old men with each other in the US for 1990, 2000 and 2010. 
The 1990 and 2000 data is from the 5\% US census. The 2010 data is from aggregating three years of the 1\% American Community Survey from 2008-2010. A state year is considered as an isolated marriage market. There were 51 states which includes DC. Individuals are distinguished by their schooling level: less than high school (L), high school graduate $(\mathrm{M})$ and university graduate $(\mathrm{H})$.

A cohabitating couple is one where a respondent answered that they are the "unmarried partner" of the head of the household.

An observation in the dataset is the number of $(r, i, j)$ relationships in a state year. Since there are three types of men and three types of women, there are potentially 9 types of matches for each type of relationship, marriage versus cohabitation.

Table 1 in Appendix C provides some summary statistics.

There are 1113 and 1283 non-zero number of cohabitations and marriages respectively. There are close to an average of 50,000 males and females of each type. The number of unmatched individuals exclude individuals whose partners are not in the $(r, i, j)$ matches considered here. For example, if a woman has a husband older than 32 , she will be counted in the number of females with her educational level and excluded in the count of the unmatched. There are close to an average of 20,000 unmatched individuals of each type. The educational distributions by types and year are in Figure 2 .

Due the small numbers of cohabitations for some observations, we reduce the effect of sampling error on our estimates by doing weighted regressions. ${ }^{10}$

Table 2 presents estimates of equation (3) by OLS. Although the OLS estimates are inconsistent, the estimates anticipate what we will find by IV. The smallest model, model 1, is in columns (1a) and (1b) where $\gamma_{i j}^{s r t}=\gamma^{r}$. The CS model, where $\alpha^{r}=\beta^{r}=\frac{1}{2}$, cannot be rejected in column 1a at the $5 \%$ significance level. But there is already evidence against the CS model in column 1b for the estimated coefficient on the unmatched females. We can also reject the hypothesis of constant return to scale (CRS), $\alpha+\beta=1$, in column $1 \mathrm{~b}$.

Model 2, in columns (2a) and (2b) add unrestricted year and match effects. The estimated year effects show that compared with 1990, the gains to cohabitation increased in 2000 and again in 2010, whereas the gains to marriage fell in 2000 and again in 2010.

Since the estimates of the match effects are difficult to interpret, we present instead the local log odds, equation (19). With three types of individuals by gender, there are four local log odds. In columns (2a) and (2b), all the local $\log$ odds are significantly positive. Thus there is strong evidence for PAM by educational attainment in both cohabitation and marriage. In fact PAM is present in both cohabitation and marriage in all our empirical models. There is mild evidence against CRS in columns (2a) and (2b).

Model 3 , in columns (3a) and (3b) add state effects to the covariates. In model 1 , the $R^{2} s$ are in the 0.5 range. The $R^{2} s$ increase to 0.9 by adding match and year effects in model 2 . The $R^{2} s$ increase to 0.92 and 0.97 in columns (3a) and $(3 \mathrm{~b})$ respectively with the addition of state effects. As a descriptive model

\footnotetext{
${ }^{10}$ Each observation is weighted by the average of $m_{i}^{s t}$ and $f_{j}^{s t}$.
} 
of marital behavior by state and year, the Cobb Douglas MMF is a very good summary of the data for these individuals.

The estimated coefficients on the unmatched increased significantly in model 3 compared with the estimates in model 1 consistent with our hypothesis that the error terms in model 1 are primarily gains to relationships effects. Since the gain to a relationship is negatively correlated with the unmatched, the estimates in model 1 are biased down relative to model 3. In model 3, CRS is easily rejected.

The test of CSPE, that $\frac{\alpha^{\mathcal{M}}}{\beta^{\mathcal{M}}} \frac{\beta^{\mathcal{C}}}{\alpha^{\mathcal{C}}}=1$, is in the second last row of the table. CSPE cannot be rejected in all three models. And in model 3, the point estimate of $\frac{\alpha^{\mathcal{M}}}{\beta \mathcal{M}} \frac{\beta^{\mathcal{C}}}{\alpha^{c}}$ is essentially 1 . As will also be the cases later, there will be no evidence against CSPE in the model with match, state and year effects by either OLS or IV.

Except for column (1a), the estimate of $\frac{\alpha}{\beta}$ is smaller than one which means that the peer effect coefficient for unmatched females, $\Phi^{0}$, is smaller than the peer coefficient for unmatched males, $\phi^{0}$. Put another way, compared with men, women's utilities as unmatched are less affected by their peers choosing to remain unmatched.

Finally in the last row of the Table 2, using lemma 2, we present the $p$-value for testing independence of the log odds of cohabitation versus marriage with respect to the sex ratio. Independence is not rejected at the $5 \%$ significance level in models 1 and 3. It is rejected in model 2. As will be shown later, these conclusions are not robust to IV estimation.

Table 3 presents IV estimates where the instruments for the unmatched, $\mu_{i 0}^{r s t}$ and $\mu_{0 j}^{r s t}$, are the population supplies $m_{i}^{r s t}$ and $f_{j}^{r s t}$. Since the error terms in the regressions represent the gains to a relationship and thus are negatively correlated with the number of unmatched, we expect the IV estimates to be larger than their OLS counterparts. Compared with their OLS counterparts, the IV estimates of the coefficients of the unmatched are marginally larger. They are significantly larger for model 3 .

The IV estimates of the local log odds and year effects are similar to their OLS counterparts. This should not be surprising. The $R^{2} s$ for the first stage regressions of the unmatched on population supplies exceed 0.95. Because the IV estimates for the unmatched are larger than the OLS estimates, the IV estimates of the constant term is smaller than their OLS counterparts.

Similar to the OLS estimates, the IV estimates for model 3 fits well. Interestingly, the estimates for $\alpha+\beta$ is close to 2 in column (3b), presenting the most suggestive evidence for the DM MMF although we can reject $\alpha=1$ or $\beta=1$. The estimates for cohabitation in column (3a) is less compelling for DM. Still, there is significant evidence for both peer and scale effects in marriage matching.

There is no evidence against CSPE. In particular in model 3 , the point estimate for $\frac{\alpha^{\mathcal{M}}}{\beta^{\mathcal{M}}} \frac{\beta^{\mathcal{C}}}{\alpha^{\mathcal{C}}}$ is again essentially 1 and the standard error is small.

The one big difference in the IV estimates compared with the OLS estimates is the test of independence of the log odds of cohabitation versus marriage with 
respect to the sex ratio. For all three models in Table 3, independence is rejected at lower than the $1 \%$ significance level.

Table 4 presents IV estimates where we allow the marriage matching patterns to change over time. Model 1 , in columns $1 \mathrm{a}$ and $1 \mathrm{~b}$, includes time varying match effects and year effects. Model 2, in columns $2 \mathrm{a}$ and $2 \mathrm{~b}$, add state effects. Unsurprisingly, based on estimates of the local log odds in 1990, PAM remains strong and significant. There is little evidence for systematic changes in the local $\log$ odds in 2000, 10 years later. There is more evidence for an increase in PAM along the main diagonal in cohabitation in 2010, 20 years later. There is also evidence of an increase in PAM in marriage between high school graduates and less than high school graduates. In general, except for a mild increase in PAM among cohabitants, there is little change in the degree of complementarity of the relationship output functions between 1990 and 2010. The stability in marriage matching pattern was anticipated in CSW and Siow. This is in strong contrast to a loss of the gains to marriage and an increase in the gains to cohabitation over the same period.

Again, there is little evidence against CSPE.

Finally, independence is rejected around the $5 \%$ significance level for model 2 and less than $1 \%$ for model 3.

In summary, model 3 in Table 3, where gains to a relationship is captured by match, state and year effects, provides a reasonable summary of marital behavior for the individuals under study. Moreover, to a first order the match effects have not changed significantly over the period of study. There is a second order increase in PAM for cohabitants. Thus analysts can focus on studying mechanisms which affected state and year effects to the gains in relationships relative to remaining unmatched. As anticipated by Fernandez-Villaverde, et. al., Adamopoulou and Drewianka, peer and scale effects in the marriage market are empirically important. Finally, independence of the log odds of the number of marriages to cohabitation with respect to the sex ratio is rejected.

\section{Conclusion}

This paper presented an easy to estimate and simulate MMF, the Cobb Douglas MMF. Several behavioral MMFs are special cases including CSPE. Empirically, we shows that peer and scale effects are quantitatively important. Independence of the log odds of the number of marriages to cohabitation with respect to the sex ratio is rejected. We also show that changes in marital matching behavior over this period are best explained by mechanisms which explain year and state effects in the gains to relationships relative to remaining unmatched.

And as we discussed in the introduction, although we are partial to CSPE and it is not rejected empirically, it should be clear that we propose the Cobb Douglas MMF precisely because we do not want to insist on a particular behavioral model of the marriage market.

In order to keep the paper within a reasonable length, our empirical study focused on a small subset of the marriageable population. Estimating the model 
on a larger subset of the population is an important agenda for future research. Also using the model to study particular mechanisms for marital change is another important topic for future research.

From an analytic perspective, we are working on sufficient conditions for characterizing increasing and/or decreasing returns to scale. It will also be useful to study other behavioral models which allow for more varied relationships between the log odds of the number of marriages to cohabitation and changes in the sex ratio.

\section{References}

Adamopoulou, Effrosyni, (2012), "Peer Effects in Young Adults' Marital Decisions", Economics Working Papers, Universidad Carlos III, Departamento de Economía

Arcidiacono, Peter, Andrew Beauchamp and Marjorie McElroy. 2010. "Terms of Endearment: An Equilibrium Model of Sex and Matching", Duke manuscript.

Becker, Gary S. "A theory of marriage: Part I." Journal of Political Economy (1973): S1-S25.

Becker, Gary S. "A Theory of Marriage: Part II." Journal of Political Economy(1974): S11-S26.

Becker, Gary Stanley. 2009. A Treatise on the Family. Harvard university press.

Blume, Lawrence E., William A. Brock, Steven N. Durlauf, and Rajshri Jayaraman. forthcoming. "Linear social interactions models." Journal of Political Economy.

Brock, William A., and Steven N. Durlauf. "Discrete choice with social interactions." Review of Economic Studies 68.2 (2001): 235-260.

Burtless, Gary. "Effects of growing wage disparities and changing family composition on the US income distribution." European Economic Review 43.4 (1999): 853-865.

Djebbari, Habiba and Bramoullé, Yann and Fortin, Bernard (2009) "Identification of peer effects through social networks" Journal of Econometrics, 150: 41-55.

Dupuy and Galichon (2012) "Personality Traits and the Marriage Market" Forthcoming in Journal of Political Economy.

Carlson, D., and T. Markham (1979): "Schur complements of diagonally dominant matrices," Czechoslovak Mathematical Journal, 29(2), 246-251.

Chiappori, Pierre-Andre and Bernard Salanié, 2014. "The Econometrics of Matching Models", Columbia University manuscript.

Chiappori, Pierre A., Bernard Salanié, and Yoram Weiss. "Partner choice and the marital college premium." (2011). Columbia University manuscript.

Choo, Eugene and Aloysius Siow. 2006a. "Who Marries Whom and Why." Journal of Political Economy 114 (1): 175-201.

Choo, Eugene and Aloysius Siow. 2006b. "Estimating a marriage matching model with spillover effects." Demography 43.3 (2006b): 463-490. 
Dagsvik, John K. "Aggregation in matching markets." International Economic Review 41.1 (2000): 27-58.

Decker, Colin, Elliott H. Lieb, Robert J. McCann, and Benjamin K. Stephens. "Unique equilibria and substitution effects in a stochastic model of the marriage market." Journal of Economic Theory 148, no. 2 (2013): 778-792.

Drewianka, Scott. (2003). "Estimating social effects in matching markets: externalities in spousal search". Review of Economics and Statistics, 85(2), 409-423.

Fernandez, Raquel, Nezih Guner, and John Knowles. 2005. "Love and Money: A Theoretical and Empirical Analysis of Household Sorting and Inequality." Quarterly Journal of Economics 120 (1): 273-344.

Fernández-Villaverde, Jesús, Jeremy Greenwood, and Nezih Guner. 2014. "From Shame to Game in One Hundred Years: An Economic Model of the Rise in Premarital Sex and its de-stigmatization." Journal of the European Economic Association 12 (1): 25-61.

Fiedler, M., and V. Ptak (1962): "On matrices with non-positive off-diagonal elements and positive principal minors", Czechoslovak Mathematical Journal, $12(3), 382-400$.

Gale, D., and H. Nikaido (159):" The Jacobian matrix and global univalence of mappings," Mathematische Annalen, 2, 81-93.

Galichon, Alfred and Salanié, Bernard, Cupid's Invisible Hand: Social Surplus and Identification in Matching Models (September 17, 2012). SSRN: http://ssrn.com/abstract $=1804623$

Galichon, Alfred, Scott Kominers and Simon Weber. In process. "Costly Concessions: Estimating the Unintended Consequences of Policy Intervention in Matching Markets."

Goldin, Claudia, and Lawrence F. Katz. "The Power of the Pill: Oral Contraceptives and Women's Career and Marriage Decisions." Journal of Political Economy 110.4 (2002).

Graham, Bryan S. "Econometric methods for the analysis of assignment problems in the presence of complementarity and social spillovers." Handbook of social economics 1 (2011): 965-1052.

Graham, Bryan S. "Comparative static and computational methods for an empirical one-to-one transferable utility matching model." Structural Econometric Models 31 (2013): 153-181.

Greenwood, Jeremy, Nazih Guner, Georgi Kocharkov, \& Cezar Santos. (2012). "Technology and the changing family: A unified model of marriage, divorce, educational attainment and married female labor-force participation". WP No. w17735. National Bureau of Economic Research.

Greenwood, Jeremy., Nazih Guner, Georgi Kocharkov, \& Cezar Santos. (2014). "Marry Your Like: Assortative Mating and Income Inequality." American Economic Review, 104(5), 348-53.

Horn, R. A., \& C. R. Johnson (2013). Matrix Analysis. Cambridge: Cambridge University Press.

Krantz, S. G., \& H. R. Parks (2003): The Implicit Function Theorem: History, Theory and Applications. Birkhauser. 
Lundberg, Shelly J., and Robert A. Pollak. "The American Family and Family Economics." Journal of Economic Perspectives 21.2 (2007): 3-26.

Manski, Charles (1993). "Identification in endogenous social effects: The reflection problem ." The Review of Economic Studies (60): 531-542.

McKenzie, L. (1959). "Matrices with dominant diagonals and economic theory." Mathematical Methods in the Social Sciences: 47-62. Stanford University Press.

Menzel, Konrad. 2014. "Large Matching Markets as Two-Sided Demand Systems", NYU manuscript.

Moffitt, Robert A., Robert Reville, and Anne E. Winkler. (1998). "Beyond single mothers: Cohabitation and marriage in the AFDC program." Demography 35.3: $259-278$.

Ismael Mourifié and Aloysius Siow (in process). "Marriage matching with frictional transfers".

Ruzhansky, M., and M. Sugimoto (2013): "On Hadamard's global inverse function theorem," Working paper.

Shi, Xiaoxia, and Matthew Shum (2014). "On Identification of a Beckerian Marriage Matching Model." California Institute of Technology manuscript.

Siow, Aloysius. forthcoming. "Testing Becker's Theory of Positive Assortative Matching." Journal of Labor Economics.

Stevenson, Betsey, and Justin Wolfers. "Marriage and Divorce: Changes and Their Driving Forces." Journal of Economic Perspectives (2007): 27-52.

Waite, Linda J., and Christine Bachrach, (2004), eds. The ties that bind: Perspectives on marriage and cohabitation. Transaction Publishers, 2004.

\section{A Existence and Uniqueness of the Matching Equilibrium}

To ease the notation, denote $\mathcal{M} \equiv a$ and $\mathcal{C} \equiv b$ in the rest of the paper. The matching equilibrium in this model is characterized by the Cobb Douglas MMF (21) and the population constraint equations

$$
\begin{array}{r}
\sum_{j=1}^{J} \mu_{i j}^{a}+\sum_{j=1}^{J} \mu_{i j}^{b}+\mu_{i 0}=m_{i}, \quad 1 \leq i \leq I \\
\sum_{i=1}^{I} \mu_{i j}^{a}+\sum_{i=1}^{I} \mu_{i j}^{b}+\mu_{0 j}=f_{j}, \quad 1 \leq j \leq J \\
\mu_{0 j}, \mu_{i 0} \geq 0, \quad 1 \leq j \leq J, 1 \leq i \leq I .
\end{array}
$$

Let $m \equiv\left(m_{1}, \ldots, m_{I}\right)^{\prime}, f \equiv\left(f_{1}, \ldots, f_{J}\right)^{\prime}, \mu \equiv\left(\mu_{10}, \ldots, \mu_{I 0}, \mu_{01}, \ldots, \mu_{0 J}\right)^{\prime}, \gamma^{r} \equiv$ $\left(\gamma_{11}^{r}, \ldots, \gamma_{1 I}^{r}, \ldots, \gamma_{I 1}^{r}, \ldots, \gamma_{I J}^{r}\right)^{\prime}$ for $r \in\{a, b\}, \beta^{r} \equiv\left(\beta_{11}^{r}, \ldots, \beta_{1 I}^{r}, \ldots, \beta_{I 1}^{r}, \ldots, \beta_{I J}^{r}\right)^{\prime}$, $\alpha^{r} \equiv\left(\alpha_{11}^{r}, \ldots, \alpha_{1 I}^{r}, \ldots, \alpha_{I 1}^{r}, \ldots, \alpha_{I J}^{r}\right)^{\prime} \beta \equiv\left(\left(\beta^{a}\right)^{\prime},\left(\beta^{b}\right)^{\prime}\right)^{\prime}, \alpha \equiv\left(\left(\alpha^{a}\right)^{\prime},\left(\alpha^{b}\right)^{\prime}\right)^{\prime}$ and $\theta \equiv\left(\left(\gamma^{a}\right)^{\prime},\left(\gamma^{b}\right)^{\prime}, \alpha^{\prime}, \beta^{\prime}\right)^{\prime}$. Let $\Gamma$ be a closed and bounded subset of $\mathbb{R}^{2 I J}$ such 
that $\theta \in \Gamma \times(0, \infty)^{2}$. Equation (21) can be written as follows:

$$
\mu_{i j}^{r}=\mu_{i 0}^{\alpha_{i j}^{r}} \mu_{0 j}^{\beta_{i j}^{r}} e^{\gamma_{i j}^{r}} \quad \text { for } r \in\{a, b\} .
$$

Now, let consider the following mapping $g:\left(\mathbb{R}_{+}^{*}\right)^{I+J} \rightarrow\left(\mathbb{R}_{+}^{*}\right)^{I+J}$

$$
\begin{array}{r}
g_{i}(\mu ; \theta)=\mu_{i 0}+\sum_{j=1}^{J} \mu_{i 0}^{\alpha_{i j}^{a}} \mu_{0 j}^{\beta_{i j}^{a}} e^{\gamma_{i j}^{a}}+\sum_{j=1}^{J} \mu_{i 0}^{\alpha_{i j}^{b}} \mu_{0 j}^{\beta_{i j}^{b}} e^{\gamma_{i j}^{b}}, \text { for } 1 \leq i \leq I, \\
g_{j+I}(\mu ; \theta)=\mu_{0 j}+\sum_{i=1}^{I} \mu_{i 0}^{\alpha_{i j}^{a}} \mu_{0 j}^{\beta_{i j}^{a}} e^{\gamma_{i j}^{a}}+\sum_{i=1}^{I} \mu_{i 0}^{\alpha_{i j}^{b}} \mu_{0 j}^{\beta_{i j}^{b}} e^{\gamma_{i j}^{b}}, \text { for } 1 \leq j \leq J .
\end{array}
$$

This mapping is obtained by just rewriting the left side of the population constraints with the Cobb Douglas MMF. We show later in Section A.1 that for every $\theta \in \Gamma \times(0, \infty)^{2} g$ is a proper mapping ${ }^{11}$ and that the Jacobian of $g(\mu ; \theta)$ (i.e. $\left.J_{g}(\mu ; \theta)\right)$ does not vanish for all $\mu$ in $\left(\mathbb{R}_{+}^{*}\right)^{I+J}$. Thus, we can invoke Hadamard's theorem ${ }^{12}$ (see Krantz and Park (2003, Theorem 6.2.8 p 126)), which tells us that $g$ is an homeomorphism i.e. (one-to-one mapping) whenever the latter two properties of $g$ hold. Then, for every $\theta \in \Gamma \times(0, \infty)^{2}$ and for all $m>0$ and $f>0$ the system of equations

$$
\begin{gathered}
\mu_{i 0}+\sum_{j=1}^{J} \mu_{i 0}^{\alpha_{i j}^{a}} \mu_{0 j}^{\beta_{i j}^{a}} e^{\gamma_{i j}^{a}}+\sum_{j=1}^{J} \mu_{i 0}^{\alpha_{i j}^{b}} \mu_{0 j}^{\beta_{i j}^{b}} e^{\gamma_{i j}^{b}}=m_{i}, \text { for } 1 \leq i \leq I \\
\mu_{0 j}+\sum_{i=1}^{I} \mu_{i 0}^{\alpha_{i j}^{a}} \mu_{0 j}^{\beta_{i j}^{a}} e^{\gamma_{i j}^{a}}+\sum_{i=1}^{I} \mu_{i 0}^{\alpha_{i j}^{b}} \mu_{0 j}^{\beta_{i j}^{b}} e^{\gamma_{i j}^{b}}=f_{j}, \text { for } 1 \leq j \leq J .
\end{gathered}
$$

admit a unique solution $0<\mu<\left(m^{\prime}, f^{\prime}\right)^{\prime}$. Therefore the equilibrium matching of the Cobb Douglas MMF model exists and is unique. The following theorem summarizes our discussion:

\section{A.1 Proof of Theorem 1}

Proof. Consider the following continuously differentiable function $g:\left(\mathbb{R}_{+}^{*}\right)^{I+J} \rightarrow$ $\left(\mathbb{R}_{+}^{*}\right)^{I+J}$

$$
\begin{array}{r}
g_{i}(\mu)=\mu_{i 0}+\sum_{j=1}^{J} \mu_{i 0}^{\alpha_{i j}^{a}} \mu_{0 j}^{\beta_{i j}^{a}} e^{\gamma_{i j}^{a}}+\sum_{j=1}^{J} \mu_{i 0}^{\alpha_{i j}^{b}} \mu_{0 j}^{\beta_{i j}^{b}} e^{\gamma_{i j}^{b}}, \\
g_{j+I}(\mu)=\mu_{0 j}+\sum_{i=1}^{I} \mu_{i 0}^{\alpha_{i j}^{a}} \mu_{0 j}^{\beta_{i j}^{a}} e^{\gamma_{i j}^{a}}+\sum_{i=1}^{I} \mu_{i 0}^{\alpha_{i j}^{b}} \mu_{0 j}^{\beta_{i j}^{b}} e^{\gamma_{i j}^{b}} .
\end{array}
$$

\footnotetext{
${ }^{11} \mathrm{~A}$ continuous function between topological spaces is called proper if the inverse images of compact subsets are compact.

${ }^{12}$ We thank Marcin Peski for pointing to us out this Hadamard's result.
} 
Notice that,

$$
\begin{aligned}
\mu_{i 0}^{\alpha_{i j}^{r}} \mu_{0 j}^{\beta_{i j}^{r}} e^{\gamma_{i j}^{r}} & =e^{\alpha_{i j}^{r} \ln \mu_{i 0}+\beta_{i j}^{r} \ln \mu_{0 j}+\gamma_{i j}^{r}} \\
& \equiv e^{\delta_{i j}^{r}}
\end{aligned}
$$

Therefore, the mapping $g(\mu)$ can be written equivalently as follows:

$$
\begin{aligned}
g_{i}(\mu) & =\mu_{i 0}+\sum_{j=1}^{J} e^{\delta_{i j}^{a}}+\sum_{j=1}^{J} e^{\delta_{i j}^{b}}, \\
g_{j+I}(\mu) & =\mu_{0 j}+\sum_{i=1}^{I} e^{\delta_{i j}^{a}}+\sum_{i=1}^{I} e^{\delta_{i j}^{b}} .
\end{aligned}
$$

Let $J_{g}(\mu)$ be the Jacobian of $g$. After a simple derivation we can show that $J_{g}(\mu)$ takes the following form:

$$
\begin{aligned}
& J_{g}(\mu)=\left(\begin{array}{ll}
\left(J_{g}\right)_{11}(\mu) & \left(J_{g}\right)_{12}(\mu) \\
\left(J_{g}\right)_{21}(\mu) & \left(J_{g}\right)_{22}(\mu)
\end{array}\right) \\
& \text { with } \\
& \left(J_{g}\right)_{11}(\mu)=\left(\begin{array}{ccc}
1+\sum_{j=1}^{J}\left[\frac{\alpha_{1 j}^{a}}{\mu_{10}} e^{\delta_{1 j}^{a}}+\frac{\alpha_{1 j}^{b}}{\mu_{10}} e^{\delta_{1 j}^{b}}\right] & \cdots & 0 \\
\vdots & \ddots & \vdots \\
0 & \cdots & 1+\sum_{j=1}^{J}\left[\frac{\alpha_{I j}^{a}}{\mu_{I 0}} e_{\delta_{I j}^{a}}^{a}+\frac{\alpha_{I j}^{b}}{\mu_{I 0}} e^{\delta_{I j}^{b}}\right]
\end{array}\right) \text {, } \\
& \left(J_{g}\right)_{12}(\mu)=\left(\begin{array}{ccc}
\frac{\beta_{11}^{a}}{\mu_{01}} e^{\delta_{11}^{a}}+\frac{\beta_{11}^{b}}{\mu_{01}} e^{\delta_{11}^{b}} & \cdots & \frac{\beta_{1 J}^{a}}{\mu_{0 J}} e^{\delta_{1 J}^{a}}+\frac{\beta_{1 J}^{b}}{\mu_{0 J}} e^{\delta_{1 J}^{b}} \\
\vdots & \ddots & \vdots \\
\frac{\beta_{I 1}^{a}}{\mu_{01}} e^{\delta_{I 1}^{a}}+\frac{\beta_{I 1}^{b}}{\mu_{01}} e^{\delta_{I 1}^{b}} & \cdots & \frac{\beta_{I J}^{a}}{\mu_{0 J}} e^{\delta_{I J}^{a}}+\frac{\beta_{I J}^{b}}{\mu_{0 J}} e^{\delta_{I J}^{b}}
\end{array}\right), \\
& \left(J_{g}\right)_{21}(\mu)=\left(\begin{array}{ccc}
\frac{\alpha_{11}^{a}}{\mu_{10}} e^{\delta_{11}^{a}}+\frac{\alpha_{11}^{b}}{\mu_{10}} e^{\delta_{11}^{b}} & \ldots & \frac{\alpha_{I 1}^{a}}{\mu_{I 0}} e^{\delta_{I 1}^{a}}+\frac{\alpha_{I 1}^{b}}{\mu_{I 0}} e^{\delta_{I 1}^{b}} \\
\vdots & \ddots & \vdots \\
\frac{\alpha_{1 J}^{a}}{\mu_{10}} e^{\delta_{1 J}^{a}}+\frac{\alpha_{1 J}^{b}}{\mu_{10}} e^{\delta_{1 J}^{b}} & \cdots & \frac{\alpha_{I J}^{a}}{\mu_{I 0}} e^{\delta_{I J}^{a}}+\frac{\alpha_{I J}^{b}}{\mu_{I 0}} e^{\delta_{I J}^{b}}
\end{array}\right), \\
& \left(J_{g}\right)_{22}(\mu)=\left(\begin{array}{ccc}
1+\sum_{i=1}^{I}\left[\frac{\beta_{i 1}^{a}}{\mu_{01}} e^{\delta_{i 1}^{a}}+\frac{\beta_{i 1}^{b}}{\mu_{01}} e^{\delta_{i 1}^{b}}\right] & \cdots & 0 \\
\vdots & \ddots & \vdots \\
0 & \cdots & 1+\sum_{i=1}^{I}\left[\frac{\beta_{i J}^{a}}{\mu_{0 J}} e^{\delta_{i J}^{a}}+\frac{\beta_{i J}^{b}}{\mu_{0 J}} e^{\delta_{i J}^{b}}\right] .
\end{array}\right) .
\end{aligned}
$$

Claim 3 The Jacobian $J_{g}(\mu)$ does not vanish for all $\mu$ in $\left(\mathbb{R}_{+}^{*}\right)^{I+J}$.

Proof. $J_{g}(\mu)$ is a column diagonally dominant matrix or diagonally dominant in the sense of McKenzie (1960) and therefore $J_{g}(\mu)$, for all $\mu>0$, is a nonsingular matrix. See McKenzie (1960, p 47-50) for more details on this result. Indeed, let us denote every element of $J_{g}(\mu), b_{k, l}$ with $1 \leq k, l \leq I+J . J_{g}(\mu)$ is diagonally dominant in the sense of McKenzie (1960) if there exist numbers $d_{l}>0$ such that $d_{l}\left|b_{l l}\right|>\sum_{k \neq l}^{I+J} d_{k}\left|b_{k l}\right|$ for $l=1, \ldots, I+J$. Here, it is sufficient 
to take $d_{l}=1$ for $1 \leq l \leq I+J$. Indeed, if you take one element in the diagonal of the matrix $\left(J_{g}\right)_{11}(\mu)$, it can be seen that this element is greater than the summation of all elements in the same column of the matrix $\left(J_{g}\right)_{21}(\mu)$.

Claim $4\left(\mathbb{R}_{+}^{*}\right)^{I+J}$ is a smooth manifold and simply connected.

Claim $5 \mathrm{~g}$ is proper.

Proof.

Definition 6 Let $\mathbb{X}, \mathbb{Y}$ be topological spaces and $g: \mathbb{X} \rightarrow \mathbb{Y}$ be a mapping. $g$ is said to be proper if whenever $K \subseteq Y$ is compact then $g^{-1}(K) \subseteq \mathbb{X}$ is compact.

Krantz and Park (2003, p 125) pointed out the following lemma that gives more operational criteria for checking if a mapping is proper.

Lemma 3 Let $U$ and $V$ be connected open sets in $\mathbb{R}^{I+J}, g: U \rightarrow V$ is a proper mapping if and only if whenever $\left\{x_{j}\right\} \subseteq U$ satisfies $x_{j} \rightarrow \partial U$ then $g\left(x_{j}\right) \rightarrow \partial V$.

Notice that $\partial V$ is used for the boundary of set $V$. Therefore, the following result completes the proof; define $\underline{\mu}=(0, \ldots, 0,0, \ldots, 0)^{\prime}$ and

$\bar{\mu}=(+\infty, \ldots,+\infty,+\infty, \ldots,+\infty)^{\prime}$. We can easily show that $\lim _{\mu \rightarrow \mu} g(\mu)=$ $(0, \ldots, 0,0, \ldots, 0)^{\prime}$ and $\lim _{\mu \rightarrow \bar{\mu}} g(\mu)=(+\infty, \ldots,+\infty,+\infty, \ldots,+\infty)^{\prime}$. $\quad$ After presenting the previous claims, we can now invoke Hadamard's theorem as stated in Krantz and Park (2003, Theroem 6.2.8 p 126). This theorem ensures that $g$ is an homeomorphism. Moreover, it is easy to see that the solution $\mu^{e q}$ of system of equations (33) satisfies the restriction $0<\mu^{e q}<\left(m^{\prime}, f^{\prime}\right)^{\prime}$. Notice that the existence of at least an equilibrium can be shown using Brouwer's fixed point theorem. Indeed, this can be done using a fixed point representation of the equilibrium of the Cobb Douglas MMF as we have done in the proof of the comparative statistics result. This completes our proof.

\section{B Comparative Statistics}

\section{B.1 Fixed point representation of the equilibrium of the Cobb Douglas MMF}

After rearranging equation (30) we have four equalities that holds for all $(i, j)$ pairs:

$$
\begin{array}{ll}
\frac{\mu_{i j}^{r}}{\mu_{i 0}}=\exp \left[\gamma_{i j}^{r}+\left(\alpha^{r}-1\right) \ln \mu_{i 0}+\beta^{r} \ln \mu_{0 j}\right] \equiv \eta_{i j}^{r} \quad \text { for } r \in\{a, b\}, \\
\frac{\mu_{i j}^{r}}{\mu_{0 j}}=\exp \left[\gamma_{i j}^{r}+\alpha^{r} \ln \mu_{i 0}+\left(\beta^{r}-1\right) \ln \mu_{0 j}\right] \equiv \zeta_{i j}^{r} \quad \text { for } r \in\{a, b\} .
\end{array}
$$


Using equations (39) and (40) we have:

$$
\begin{array}{ll}
\sum_{j=1}^{J} \mu_{i j}^{a}+\sum_{j=1}^{J} \mu_{i j}^{b}=\mu_{i 0} \sum_{j=1}^{J}\left[\eta_{i j}^{a}+\eta_{i j}^{b}\right], & 1 \leq i \leq I, \\
\sum_{i=1}^{I} \mu_{i j}^{a}+\sum_{i=1}^{I} \mu_{i j}^{b}=\mu_{0 j} \sum_{i=1}^{I}\left[\zeta_{i j}^{a}+\zeta_{i j}^{b}\right], & 1 \leq j \leq J .
\end{array}
$$

Manipulating the population constraints (28), (29) we have the following:

$$
\begin{gathered}
\mu_{i 0}=\frac{m_{i}}{1+\sum_{j=1}^{J}\left[\eta_{i j}^{a}+\eta_{i j}^{b}\right]} \equiv B_{i 0}, \quad 1 \leq i \leq I \\
\mu_{0 j}=\frac{f_{j}}{1+\sum_{i=1}^{I}\left[\zeta_{i j}^{a}+\zeta_{i j}^{b}\right]} \equiv B_{0 j}, \quad 1 \leq j \leq J .
\end{gathered}
$$

Let $B(\mu ; m, f, \theta) \equiv\left(B_{10}(.), \ldots, B_{I 0}(.), B_{01}(.), \ldots, B_{0 J}(.)\right)^{\prime}$. For a fixed $\theta$ we have shown that the $(I+J)$ vector $\mu$ of the number of agents of each type who choose not to match is a solution to $(I+J)$ vector of implicit functions

$$
\mu-B(\mu ; m, f, \theta)=0
$$

Let $\mathbb{T}_{\epsilon}=\left\{\epsilon \leq \mu_{10} \leq m_{1}, \ldots, \epsilon \leq \mu_{I 0} \leq m_{I}, \epsilon \leq \mu_{01} \leq f_{1}, \ldots, \epsilon \leq \mu_{0 J} \leq f_{J}\right\}$ be a closed and bounded rectangular region in $\mathbb{R}^{I+J}$ with $\epsilon$ some arbitrarily small positive constant. We know from Theorem 1 that the fixed point representation has a unique solution $\mu^{e q}>0$. We can verify that $\mu^{e q} \in \mathbb{T}_{\epsilon}$. Now, let $J(\mu)=$ $I_{I+J}-\nabla_{\mu} B(\mu ; m, f, \theta)$ with $\nabla_{\mu} B(\mu ; m, f, \theta)=\frac{\partial B(\mu ; m, f, \theta)}{\partial \mu^{\prime}}$ be the $(I+J) \times(I+J)$ Jacobian matrix associated with (44). For a fixed $\theta$ we have shown that the $(I+J)$ vector $\mu$ of the number of agents of each type who choose not to match is a solution to $(I+J)$ vector of implicit functions

$$
\mu-B(\mu ; m, f, \theta)=0
$$

\section{B.2 Proof of Theorem 2}

All derivation in this section will be done at the matching equilibrium $\mu^{e q}$. However, to ease notation we will use the notation $\mu$.

Proof.

Step 0: Derivation of the $J(\mu)$ matrix.

To ease the notation, in the following we will use $B(\mu)$ to denote $B(\mu ; m, f, \theta)$ whenever no confusion is possible.

$J(\mu)=I_{I+J}-\nabla_{\mu} B(\mu)$. After tedious but simple manipulations we can show that

$\nabla_{\mu} B(\mu)=\left(\begin{array}{ll}E_{11}(\mu) & E_{12}(\mu) \\ E_{21}(\mu) & E_{22}(\mu)\end{array}\right)$

with

$E_{11}(\mu)=\operatorname{diag}\left\{\sum_{j=1}^{J} e_{j \mid 1}(\mu), \ldots, \sum_{j=1}^{J} e_{j \mid I}(\mu)\right\}$, 


$$
\begin{aligned}
& E_{22}(\mu)=\operatorname{diag}\left\{\sum_{i=1}^{I} g_{i \mid 1}(\mu), \ldots, \sum_{i=1}^{I} g_{i \mid J}(\mu)\right\} \text { where } \\
& e_{j \mid i}=\frac{m_{i}}{\mu_{i 0}}\left[\frac{\left(1-\alpha^{a}\right) \eta_{i j}^{a}+\left(1-\alpha^{b}\right) \eta_{i j}^{b}}{\left(1+\sum_{j=1}^{J}\left[\eta_{i j}^{a}+\eta_{i j}^{b}\right]\right)^{2}}\right], g_{i \mid j}=\frac{f_{j}}{\mu_{0 j}}\left[\frac{\left(1-\beta^{a}\right) \zeta_{i j}^{a}+\left(1-\beta^{b}\right) \zeta_{i j}^{b}}{\left(1+\sum_{i=1}^{I}\left[\zeta_{i j}^{a}+\zeta_{i j}^{b}\right]\right)^{2}}\right] . \\
& E_{12}(\mu)=-\left(\begin{array}{ccc}
\frac{\mu_{10}}{\mu_{01}} \hat{e}_{1 \mid 1} & \cdots & \frac{\mu_{10}}{\mu_{0 J}} \hat{e}_{J \mid 1} \\
\vdots & \ddots & \vdots \\
\frac{\mu_{I 0}}{\mu_{01}} \hat{e}_{1 \mid I} & \cdots & \frac{\mu_{I 0}}{\mu_{0 J}} \hat{e}_{J \mid I}
\end{array}\right), E_{21}(\mu)=-\left(\begin{array}{ccc}
\frac{\mu_{01}}{\mu_{10}} \hat{g}_{1 \mid 1} & \cdots & \frac{\mu_{01}}{\mu_{I 0}} \hat{g}_{I \mid 1} \\
\vdots & \ddots & \vdots \\
\frac{\mu_{0 J}}{\mu_{10}} \hat{g}_{1 \mid J} & \cdots & \frac{\mu_{0 J}}{\mu_{I 0}} \hat{g}_{I \mid J}
\end{array}\right)
\end{aligned}
$$

where

$\hat{e}_{j \mid i}=\frac{m_{i}}{\mu_{0 j}}\left[\frac{\beta^{a} \eta_{i j}^{a}+\beta^{b} \eta_{i j}^{b}}{\left(1+\sum_{j=1}^{J}\left[\eta_{i j}^{a}+\eta_{i j}^{a}\right]\right)^{2}}\right], \hat{g}_{i \mid j}=\frac{f_{j}}{\mu_{i 0}}\left[\frac{\alpha^{a} \zeta_{i j}^{a}+\alpha^{b} \zeta_{i j}^{b}}{\left(1+\sum_{i=1}^{I}\left[\zeta_{i j}^{a}+\zeta_{i j}^{b}\right]\right)^{2}}\right]$.

Now, it is important to remark that at the equilibrium when (44) holds, we get simplified versions of $e_{j \mid i}, g_{i \mid j}, \hat{e}_{j \mid i}$, and $\hat{g}_{i \mid j}$ which are the following:

$$
\begin{aligned}
& e_{j \mid i}=\frac{\left(1-\alpha^{a}\right) \eta_{i j}^{a}+\left(1-\alpha^{b}\right) \eta_{i j}^{b}}{1+\sum_{j=1}^{J}\left[\eta_{i j}^{a}+\eta_{i j}^{b}\right]}=\frac{1}{m_{i}}\left[\left(1-\alpha^{a}\right) \mu_{i j}^{a}+\left(1-\alpha^{b}\right) \mu_{i j}^{b}\right] ; \\
& g_{j \mid i}=\frac{\left(1-\beta^{a}\right) \zeta_{i j}^{a}+\left(1-\beta^{b}\right) \zeta_{i j}^{b}}{1+\sum_{i=1}^{I}\left[\zeta_{i j}^{a}+\zeta_{i j}^{b}\right]}=\frac{1}{f_{j}}\left[\left(1-\beta^{a}\right) \mu_{i j}^{a}+\left(1-\beta^{b}\right) \mu_{i j}^{b}\right] ; \\
& \hat{e}_{j \mid i}=\frac{\beta^{a} \eta_{i j}^{a}+\beta^{b} \eta_{i j}^{b}}{1+\sum_{j=1}^{J}\left[\eta_{i j}^{a}+\eta_{i j}^{b}\right]}=\frac{1}{m_{i}}\left[\beta^{a} \mu_{i j}^{a}+\beta^{b} \mu_{i j}^{b}\right] ; \\
& \hat{g}_{j \mid i}=\frac{\alpha^{a} \zeta_{i j}^{a}+\alpha^{b} \zeta_{i j}^{b}}{1+\sum_{i=1}^{I}\left[\zeta_{i j}^{a}+\zeta_{i j}^{b}\right]}=\frac{1}{f_{j}}\left[\alpha^{a} \mu_{i j}^{a}+\alpha^{b} \mu_{i j}^{b}\right] ;
\end{aligned}
$$

An appropriate adaptation of the supplement calculation of Graham (2013) (not published) would help the reader to understand some details of the calculations, that we have done here. Note that $0<\sum_{j=1}^{J} e_{j \mid i}(\mu)<1$, for all $1 \leq i \leq I$, and $0<\sum_{i=1}^{I} g_{i \mid j}(\mu)<1$ for all $1 \leq j \leq J$ whenever $0<\beta^{r}<1$ and $0<\alpha^{r}<1$ for $r \in\{a, b\}$. Now, we can write $J(\mu)$ at the equilibrium. We have the following:

$J(\mu)=\left(\begin{array}{ll}J_{11}(\mu) & J_{12}(\mu) \\ J_{21}(\mu) & J_{22}(\mu)\end{array}\right)$

where $J_{11}(\mu)=I\{I\}-E_{11}(\mu), J_{22}(\mu)=I\{J\}-E_{22}(\mu), J_{12}(\mu)=-E_{12}(\mu)$, $J_{21}(\mu)=-E_{21}(\mu)$

Step 1: Factorization of the $J(\mu)$ matrix

Recall $J(\mu)=\left(\begin{array}{ll}J_{11}(\mu) & J_{12}(\mu) \\ J_{21}(\mu) & J_{22}(\mu)\end{array}\right)$, where

$J_{12}(\mu)=\operatorname{diag}(m)^{-1}\left\{\beta^{a}\left(\begin{array}{ccc}\frac{\mu_{10}}{\mu_{01}} \mu_{11}^{a} & \cdots & \frac{\mu_{10}}{\mu_{0 J}} \mu_{1 J}^{a} \\ \vdots & \ddots & \vdots \\ \frac{\mu_{I 0}}{\mu_{01}} \mu_{I 1}^{a} & \cdots & \frac{\mu_{I 0}}{\mu_{0 J}} \mu_{I J}^{a}\end{array}\right)+\beta^{b}\left(\begin{array}{ccc}\frac{\mu_{10}}{\mu_{01}} \mu_{11}^{b} & \cdots & \frac{\mu_{10}}{\mu_{0 J}} \mu_{1 J}^{b} \\ \vdots & \ddots & \vdots \\ \frac{\mu_{I 0}}{\mu_{01}} \mu_{I 1}^{b} & \cdots & \frac{\mu_{I 0}}{\mu_{0 J}} \mu_{I J}^{b}\end{array}\right)\right\}$

Define $\operatorname{diag}\left(\mu_{.0}\right)=\operatorname{diag}\left(\mu_{10}, \ldots, \mu_{I 0}\right), \operatorname{diag}\left(\mu_{0 .}\right)=\operatorname{diag}\left(\mu_{01}, \ldots, \mu_{0 J}\right)$ and $R^{r}=$

$$
\begin{aligned}
& \left(\begin{array}{ccc}
\mu_{11}^{r} & \cdots & \mu_{1 J}^{r} \\
\vdots & \ddots & \vdots \\
\mu_{I 1}^{r} & \cdots & \mu_{I J}^{r}
\end{array}\right) \text { Therefore, } \\
& \quad J_{12}(\mu)=\operatorname{diag}\left(\mu_{.0}\right) \operatorname{diag}(m)^{-1}\left[\beta^{a} R^{a}+\beta^{b} R^{b}\right] \operatorname{diag}\left(\mu_{0 .}\right)^{-1}
\end{aligned}
$$

Similarly, we can show that $J_{21}(\mu)$ can be factored as follows:

$$
J_{21}(\mu)=\operatorname{diag}\left(\mu_{0 .}\right) \operatorname{diag}(f)^{-1}\left[\alpha^{a}\left(R^{a}\right)^{\prime}+\alpha^{b}\left(R^{b}\right)^{\prime}\right] \operatorname{diag}\left(\mu_{.0}\right)^{-1}
$$


We also factor also $J_{11}(\mu)$ and $J_{22}(\mu)$ as follows:

$$
\begin{gathered}
J_{11}(\mu)=I_{I}-\operatorname{diag}(m)^{-1}\left[\left(1-\alpha^{a}\right) R_{\iota J}^{a}+\left(1-\alpha^{a}\right) R_{\iota J}^{b}\right], \\
J_{22}(\mu)=I_{J}-\operatorname{diag}(f)^{-1}\left[\left(1-\beta^{a}\right)\left(R^{a}\right)_{\iota I}^{\prime}+\left(1-\beta^{b}\right)\left(R^{b}\right)_{\iota I}^{\prime}\right] .
\end{gathered}
$$

where $R_{\iota J}^{r}=\left(\sum_{j=1}^{J} \mu_{1 j}^{r}, \ldots, \sum_{j=1}^{J} \mu_{I j}^{r}\right)^{\prime}$ and $\left(R^{r}\right)_{\iota I}=\left(\sum_{i=1}^{I} \mu_{i 1}^{r}, \ldots, \sum_{i=1}^{I} \mu_{i J}^{r}\right)$.

After rearranging we can show that:

$$
J(\mu)=C(\mu)^{-1}\left[A(\mu)+U(\mu) B_{0}(\mu) U(\mu)^{-1}\right]
$$

where

$$
\begin{aligned}
& C(\mu)=\left(\begin{array}{cc}
\operatorname{diag}(m) & 0 \\
0 & \operatorname{diag}(f)
\end{array}\right) \\
& A(\mu)=\left(\begin{array}{cc}
\operatorname{diag}\left(m-\left(1-\alpha^{a}\right) R_{\iota J}^{a}-\left(1-\alpha^{b}\right) R_{\iota J}^{b}\right) & \operatorname{diag}\left(f-\left(1-\beta^{a}\right)\left(R^{a}\right)_{\iota I}^{\prime}-\left(1-\beta^{b}\right)\left(R^{b}\right)_{\iota I}^{\prime}\right)
\end{array}\right) \\
& U(\mu)=\left(\begin{array}{cc}
\operatorname{diag}\left(\mu_{.0}\right) & 0 \\
0 & \operatorname{diag}\left(\mu_{0 .}\right)
\end{array}\right) \\
& B_{0}(\mu)=\left(\begin{array}{cc}
0 & \beta^{a} R^{a}+\beta^{b} R^{b} \\
\alpha^{a}\left(R^{a}\right)^{\prime}+\alpha^{b}\left(R^{b}\right)^{\prime} & 0)
\end{array}\right) .
\end{aligned}
$$

Therefore, $J(\mu)$ can be equivalently rewritten as:

$$
\begin{aligned}
J(\mu) & =U(\mu) C(\mu)^{-1}\left[A(\mu)+B_{0}(\mu)\right] U(\mu)^{-1} \\
& =U(\mu) H(\mu) U(\mu)^{-1}
\end{aligned}
$$

where

$$
H(\mu)=C(\mu)^{-1}\left[A(\mu)+B_{0}(\mu)\right] .
$$

- Let us write $H(\mu)$ in detail:

$H(\mu)=\left(\begin{array}{ll}H_{11}(\mu) & H_{12}(\mu) \\ H_{21}(\mu) & H_{22}(\mu)\end{array}\right)$

with

$H_{11}(\mu)=\left(\begin{array}{ccc}1-\frac{\sum_{j=1}^{J}\left[\left(1-\alpha^{a}\right) \mu_{1 j}^{a}+\left(1-\alpha^{b}\right) \mu_{1 j}^{b}\right]}{m_{1}} & \cdots & 0 \\ \vdots & \ddots & \vdots \\ 0 & \cdots & 1-\frac{\sum_{j=1}^{J}\left[\left(1-\alpha^{a}\right) \mu_{I j}^{a}+\left(1-\alpha^{b}\right) \mu_{I j}^{b}\right]}{m_{I}}\end{array}\right)$,

$H_{12}(\mu)=\left(\begin{array}{ccc}\frac{\beta^{a} \mu_{11}^{a}+\beta^{b} \mu_{11}^{b}}{m_{1}} & \ldots & \frac{\beta^{a} \mu_{1 J}^{a}+\beta^{b} \mu_{1 J}^{b}}{m_{1}} \\ \vdots & \ddots & \vdots \\ \frac{\beta^{a} \mu_{I 1}^{a}+\beta^{b} \mu_{I 1}^{b}}{m_{I}} & \ldots & \frac{\beta^{a} \mu_{I J}^{a}+\beta^{b} \mu_{I J}^{b}}{m_{I}}\end{array}\right), H_{21}(\mu)=\left(\begin{array}{ccc}\frac{\alpha^{a} \mu_{11}^{a}+\alpha^{b} \mu_{11}^{b}}{f_{1}} & \ldots & \frac{\alpha^{a} \mu_{I 1}^{a}+\alpha^{b} \mu_{I 1}^{b}}{f_{1}} \\ \vdots & \ddots & \vdots \\ \frac{\alpha^{a} \mu_{1 J}^{a}+\alpha^{b} \mu_{1 J}^{b}}{f_{J}} & \ldots & \frac{\alpha^{a} \mu_{I J}^{a}+\alpha^{b} \mu_{I J}^{b}}{f_{J}}\end{array}\right)$,

$H_{22}(\mu)=\left(\begin{array}{ccc}1-\frac{\sum_{i=1}^{I}\left[\left(1-\beta^{a}\right) \mu_{i 1}^{a}+\left(1-\beta^{b}\right) \mu_{i 1}^{b}\right]}{m_{1}} & \cdots & 0 \\ \vdots & \ddots & \vdots \\ 0 & \cdots & 1-\frac{\sum_{i=1}^{I}\left[\left(1-\beta^{a}\right) \mu_{i J J}^{a}+\left(1-\beta^{b}\right) \mu \alpha^{b} b_{i J}\right]}{f_{J}}\end{array}\right)$.

Similar to Graham (2013, p 16), we observe that all elements of $H(\mu)$ are nonnegative whenever $0<\beta^{r} ; \alpha^{r} \leq 1$.

Step 2: Derivation of M-matrix property 
The main goal of this step is to show that the Schur complements of $H(\mu)$ the upper $I \times I\left(H_{11}\right)$ and lower $J \times J\left(H_{22}\right)$ diagonal blocks, (i.e. $S H_{11}=$ $H_{22}-H_{21} H_{11}^{-1} H_{12}$ and $\left.S H_{22}=H_{11}-H_{12} H_{22}^{-1} H_{21}\right)$ are M-matrices which implies $S H_{11}^{-1} \geqq 0$ and $S H_{22}^{-1} \geqq 0$. To show that, we first need to show that $H(\mu)$ is row diagonally dominant. In other terms, if we denote the element of $H(\mu), h_{i j}$ with $1 \leq i, j \leq I+J$ we need to show that there exist $d_{i}>0$ such that $d_{i}\left|h_{i i}\right|>\sum_{j \neq i}^{I+J} d_{j}\left|h_{i j}\right|$. This will be difficult to show without further restrictions on $\beta^{r}$ and $\alpha^{r}$. Graham $(2013, \mathrm{p} 15)$ showed this result in the particular case where the two following restrictions hold simultaneously: $\beta^{r}+\alpha^{r}=1$ and $\beta^{a}=\beta^{b}$. Here, we will impose some conditions on the coefficients $\beta^{r}$ and $\alpha^{r}$ that ensure $H(\mu)$ to be row diagonally dominant. Let first assume that $0<\beta^{r} ; \alpha^{r}<1$, then $h_{i j} \geq 0$ for $1 \leq i, j \leq I+J$.

Case 1: $1 \leq i \leq I$

$$
\left|h_{i i}\right|>\sum_{j \neq i}^{I+J}\left|h_{i j}\right| \Leftrightarrow \sum_{j=1}^{J}\left(\left(1-\alpha^{a}+\beta^{a}\right) \mu_{i j}^{a}+\left(1-\alpha^{b}+\beta^{b}\right) \mu_{i j}^{b}\right)<m_{i}(
$$

Notice that

$$
\begin{array}{r}
\max \left(\left(1-\alpha^{a}+\beta^{a}\right),\left(1-\alpha^{b}+\beta^{b}\right)\right) \sum_{j=1}^{J}\left(\mu_{i j}^{a}+\mu_{i j}^{b}\right)<m_{i} \Rightarrow \\
\sum_{j=1}^{J}\left(\left(1-\alpha^{a}+\beta^{a}\right) \mu_{i j}^{a}+\left(1-\alpha^{b}+\beta^{b}\right) \mu_{i j}^{b}\right)<m_{i},
\end{array}
$$

and

$$
\begin{gathered}
\max \left(\left(1-\alpha^{a}+\beta^{a}\right),\left(1-\alpha^{b}+\beta^{b}\right)\right) \sum_{j=1}^{J}\left(\mu_{i j}^{a}+\mu_{i j}^{b}\right)<m_{i} \quad \Leftrightarrow \\
\max \left(\left(1-\alpha^{a}+\beta^{a}\right),\left(1-\alpha^{b}+\beta^{b}\right)\right) \rho_{i}^{m}<1,
\end{gathered}
$$

where $\rho_{i}^{m} \equiv \frac{m_{i}-\mu_{i 0}}{m_{i}}$ is the rate of matched men of type $i$. The latter inequality is equivalent to $\max \left(\beta^{b}-\alpha^{b}, \beta^{a}-\alpha^{a}\right)<\frac{1-\rho_{i}^{m}}{\rho_{i}^{m}}$. Therefore, if $\max \left(\beta^{b}-\alpha^{b}, \beta^{a}-\right.$ $\left.\alpha^{a}\right)<\frac{1-\rho_{i}^{m}}{\rho_{i}^{m}}$ for all $i$ then $\left|h_{i i}\right|>\sum_{j \neq i}^{I+J}\left|h_{i j}\right|$.

Case 2: $I+1 \leq i \leq I+J$.

Similarly, we can show that if $\min \left(\beta^{b}-\alpha^{b}, \beta^{a}-\alpha^{a}\right)>-\frac{1-\rho_{j}^{f}}{\rho_{j}^{f}}$ for all $j$ where $\rho_{j}^{f} \equiv \frac{f_{j}-\mu_{0 j}}{f_{j}}$ is the rate of matched women of type $j$, then we have $\left|h_{i i}\right|>$ $\sum_{j \neq i}^{I+J}\left|h_{i j}\right|$.

Assume that the two latter restrictions on $\beta^{r}$ and $\alpha^{r}$ hold in the rest of the proof. The Schur complements of the $H(\mu)$ upper $I \times I$ and lower $J \times J$ diagonal blocks are $S H_{11}=H_{22}-H_{21}\left(H_{11}\right)^{-1} H_{12}$ and $S H_{22}=H_{11}-H_{12}\left(H_{22}\right)^{-1} H_{21}$. Since $H$ has been showed to be diagonally dominant, Theorem 1 of Carlson and 
Markham (1979 p 249) implies that the two schur complements are also diagonally dominant. Therefore, $S H_{11}$ and $S H_{22}$ are also row diagonally dominant. We can easily see that $S H_{11}$ and $S H_{22}$ are also $Z$-matrices (i.e., members of the class of real matrices with nonpositive off-diagonal elements). By applying Theorem 4.3 of Fiedler and Ptak (1962) it follows that they are M-matrices and then $S H_{11}^{-1} \geqq 0$ and $S H_{22}^{-1} \geqq 0$. These results are sufficient to establish the sign structure of $H^{-1}(\mu) . H^{-1}(\mu)=\left(\begin{array}{ll}W_{11} & W_{12} \\ W_{21} & W_{22}\end{array}\right)=\left(\begin{array}{ccc}+ & \vdots & - \\ \cdots & & \ldots \\ - & \vdots & +\end{array}\right)$ where $W_{i j}$ are exactly defined as defined in Graham (2013. p 16).

Step 3: Derivation of $H^{-1}(\mu)$

Following Graham we can show the following inequalities:

$$
\begin{aligned}
& W_{11} \geq H_{11}^{-1}+H_{11}^{-1} H_{12} H_{22}^{-1} H_{21} H_{11}^{-1}=L W_{11} \\
& W_{22} \geq H_{22}^{-1}+H_{22}^{-1} H_{21} H_{11}^{-1} H_{12} H_{22}^{-1}=L W_{22} \\
& W_{12} \leq-H_{11}^{-1} H_{12} H_{22}^{-1}=U W_{12} \\
& W_{21} \leq-H_{22}^{-1} H_{21} H_{11}^{-1}=U W_{21} .
\end{aligned}
$$

Using the expression of the matrix $H(\mu)$ and after some tedious calculations we can show the following: $L W_{11}=H_{11}^{-1}+$

$$
\left(\begin{array}{cccc}
\frac{1}{m_{1}^{*}} \frac{m_{1}}{m_{1}^{*}} \sum_{j=1}^{J} \frac{\left[\alpha^{a} \mu_{1 j}^{a}+\alpha^{b} \mu_{1 j}^{b}\right]\left[\beta^{a} \mu_{1 j}^{a}+\beta^{b} \mu_{1 j}^{b}\right]}{f_{j}^{*}} & \cdots & \frac{1}{m_{1}^{*}} \frac{m_{I}}{m_{I}^{*}} \sum_{j=1}^{J} \frac{\left[\alpha^{a} \mu_{I j}^{a}+\alpha^{b} \mu_{I j}^{b}\right]\left[\beta^{a} \mu_{1 j}^{a}+\beta^{b} \mu_{1 j}^{b}\right]}{f_{j}^{*}} \\
\vdots & \ddots & \vdots \\
\frac{1}{m_{I}^{*}} \frac{m_{1}}{m_{1}^{*}} \sum_{j=1}^{J} \frac{\left[\alpha^{a} \mu_{1 j}^{a}+\alpha^{b} \mu_{1 j}^{b}\right]\left[\beta^{a} \mu_{I j}^{a}+\beta^{b} \mu_{I j}^{b}\right]}{f_{j}^{*}} & \cdots & \frac{1}{m_{I}^{*}} \frac{m_{I}}{m_{I}^{*}} \sum_{j=1}^{J} \frac{\left[\alpha^{a} \mu_{I j}^{a}+\alpha^{b} \mu_{I I}^{b}\right]\left[\beta^{a} \mu_{I j}^{a}+\beta^{b} \mu_{I j}^{b}\right]}{f_{j}^{*}}
\end{array}\right)
$$

where

$$
m_{i}^{*} \equiv m_{i}-\sum_{j=1}^{J}\left[\left(1-\alpha^{a}\right) \mu_{i j}^{a}+\left(1-\alpha^{b}\right) \mu_{i j}^{b}\right], \text { for all } 1 \leq i \leq I
$$

and

$$
f_{j}^{*} \equiv f_{j}-\sum_{i=1}^{I}\left[\left(1-\beta^{a}\right) \mu_{i j}^{a}+\left(1-\beta^{b}\right) \mu_{i j}^{b}\right], \text { for all } 1 \leq j \leq J .
$$

Moreover, we can show that:

$$
\begin{aligned}
\left(L W_{11}\right)_{i i} & =\frac{m_{i}}{m_{i}^{*}}\left[1+\frac{1}{m_{i}^{*}} \sum_{j=1}^{J} \frac{\left[\alpha^{a} \mu_{i j}^{a}+\alpha^{b} \mu_{i j}^{b}\right]\left[\beta^{a} \mu_{i j}^{a}+\beta^{b} \mu_{i j}^{b}\right]}{f_{j}^{*}}\right] \\
& >1
\end{aligned}
$$

for all $1 \leq i \leq I$. Therefore we have $L W_{11}>I_{I}$. Similarly, we have also the following:

$L W_{22}=H_{22}^{-1}+$ 


$$
\begin{aligned}
& \left(\begin{array}{cccc}
\frac{1}{f_{1}^{*}} \frac{f_{1}}{f_{1}^{*}} \sum_{i=1}^{I} \frac{\left[\alpha^{a} \mu_{i 1}^{a}+\alpha^{b} \mu_{i 1}^{b}\right]\left[\beta^{a} \mu_{i 1}^{a}+\beta^{b} \mu_{i 1}^{b}\right]}{m_{i}^{*}} & \cdots & \frac{1}{f_{1}^{*}} \frac{f_{J}}{f_{J}^{*}} \sum_{i=1}^{I} \frac{\left[\alpha^{a} \mu_{i 1}^{a}+\alpha^{b} \mu_{i 1}^{b}\right]\left[\beta^{a} \mu_{i J}^{a}+\beta^{b} \mu_{i J}^{b}\right]}{m_{i}^{*}} \\
\vdots & \ddots & \vdots \\
\frac{1}{f_{J}^{*}} \frac{f_{1}}{f_{1}^{*}} \sum_{i=1}^{I} \frac{\left[\alpha^{a} \mu_{i J}^{a}+\alpha^{b} \mu_{i J}^{b}\right]\left[\beta^{a} \mu_{i 1}^{a}+\beta^{b} \mu_{i 1}^{b}\right]}{m_{i}^{*}} & \cdots & \frac{1}{f_{J}^{*}} \frac{f_{J}}{f_{J}^{*}} \sum_{i=1}^{I} \frac{\left[\alpha^{a} \mu_{i J}^{a}+\alpha^{b} \mu_{i J}^{b}\right]\left[\beta^{a} \mu_{i J}^{a}+\beta^{b} \mu_{i J}^{b}\right]}{m_{i}^{*}}
\end{array}\right) \\
& \text { Moreover, we can show that: } \\
&
\end{aligned}
$$

$$
\begin{aligned}
\left(L W_{22}\right)_{j j} & =\frac{f_{j}}{f_{j}^{*}}\left[1+\frac{1}{f_{j}^{*}} \sum_{i=1}^{I} \frac{\left[\alpha^{a} \mu_{i j}^{a}+\alpha^{b} \mu_{i j}^{b}\right]\left[\beta^{a} \mu_{i j}^{a}+\beta^{b} \mu_{i j}^{b}\right]}{m_{i}^{*}}\right] \\
& >1
\end{aligned}
$$

for all $1 \leq j \leq J$. Therefore, we have $L W_{11}>I_{J}$. Now, let us look at the off-diagonal blocks of $H(\mu)^{-1}$.

$$
\begin{aligned}
U W_{12}=-\left(\begin{array}{ccc}
\frac{\left[\beta^{a} \mu_{11}^{a}+\beta^{b} \mu_{11}^{b}\right]}{m_{1}^{*} f_{1}^{*}} f_{1} & \cdots & \frac{\left[\beta^{a} \mu_{1 J}^{a}+\beta^{b} \mu_{1 J}^{b}\right]}{m_{1}^{*} f_{J}^{*}} f_{J} \\
\vdots & \ddots & \vdots \\
\frac{\left[\beta^{a} \mu_{I 1}^{a}+\beta^{b} \mu_{I 1}^{b}\right]}{m_{I}^{*} f_{1}^{*}} f_{1} & \cdots & \frac{\left[\beta^{a} \mu_{I J}^{a}+\beta^{b} \mu_{I J}^{b}\right]}{m_{I}^{*} f_{J}^{*}} f_{J}
\end{array}\right) \\
\text { and } U W_{21}=-\left(\begin{array}{ccc}
\frac{\left[\alpha^{a} \mu_{11}^{a}+\alpha^{b} \mu_{11}^{b}\right]}{m_{1}^{*} f_{1}^{*}} m_{1} & \cdots & \frac{\left[\alpha^{a} \mu_{I 1}^{a}+\alpha^{b} \mu_{I 1}^{b}\right]}{m_{1}^{*} f_{J}^{*}} m_{I} \\
\vdots & \ddots & \vdots \\
\frac{\left[\alpha^{a} \mu_{1 J}^{a}+\alpha^{b} \mu_{1 J}^{b}\right]}{m_{I}^{*} f_{1}^{*}} m_{1} & \cdots & \frac{\left[\alpha^{a} \mu_{I J}^{a}+\alpha^{b} \mu_{I J}^{b}\right]}{m_{I}^{*} f_{J}^{*}} m_{I}
\end{array}\right)
\end{aligned}
$$

\section{Step 4: Main results}

\section{Case 1: Type specific elasticities of single hood}

By applying the implicit function theorem to the equation (44) we have: $\frac{\partial \mu}{\partial m_{i}}=$ $J(\mu)^{-1} \frac{\partial B}{\partial m_{i}}$ for $1 \leq i \leq I$ and $\frac{\partial \mu}{\partial f_{j}}=J(\mu)^{-1} \frac{\partial B}{\partial f_{j}}$ for all $1 \leq j \leq J$, where $\frac{\partial B}{\partial m_{i}}=\left(0, \ldots, 0, \frac{\mu_{i 0}}{m_{i}}, 0, \ldots, 0\right)^{\prime}$ and $\frac{\partial B}{\partial f_{j}}=\left(0, \ldots, 0, \frac{\mu_{0 j}}{f_{j}}, 0, \ldots, 0\right)^{\prime}$ are $(I+J)$ vectors such that the non-zero entries are respectively at the $i^{\text {th }}$ row and the $(I+j)^{t h}$ row. Let $h_{k}=(0, \ldots, 0,1,0, \ldots, 0)^{\prime}$ be a $(I+J)$ vector such that the non-zero entry is at the $k^{t h}$ row. We have the following:

$$
\begin{aligned}
U(\mu)^{-1} \frac{\partial \mu}{\partial m_{i}} m_{i} & =U(\mu)^{-1} J(\mu)^{-1} \frac{\partial B}{\partial m_{i}} m_{i} \\
& =H(\mu)^{-1} U(\mu)^{-1} h_{i} \mu_{i 0} \\
& =H(\mu)^{-1} h_{i} \\
& =\left[H(\mu)^{-1}\right] \cdot i
\end{aligned}
$$

for $1 \leq i \leq I$, where $\left[H(\mu)^{-1}\right]_{. i}$ represents the $i^{t h}$ column of the matrix $H(\mu)^{-1}$. Similarly, we can show that $U(\mu)^{-1} \frac{\partial \mu}{\partial f_{j}} f_{j}=\left[H(\mu)^{-1}\right]_{\cdot(I+j)}$ for $1 \leq j \leq J$. Putting these results together, we get the following inequalities:

$\frac{m_{i}}{\mu_{k 0}} \frac{\partial \mu_{k 0}}{\partial m_{i}} \geq\left\{\begin{array}{ll}\frac{1}{m_{i}^{*}} \frac{m_{k}}{m_{k}^{*}} \sum_{j=1}^{J} \frac{\left[\alpha^{a} \mu_{k j}^{a}+\alpha^{b} \mu_{k j}^{b}\right]\left[\beta^{a} \mu_{k j}^{a}+\beta^{b} \mu_{k j}^{b}\right]}{f_{j}^{*}}>0 & \text { if } k \neq i \\ \frac{m_{i}}{m_{i}^{*}}\left[1+\frac{1}{m_{i}^{*}} \sum_{j=1}^{J} \frac{\left[\alpha^{a} \mu_{i j}^{a}+\alpha^{b} \mu_{i j}^{b}\right]\left[\beta^{a} \mu_{i j}^{a}+\beta^{b} \mu_{i j}^{b}\right]}{f_{j}^{*}}\right]>1 & \text { if } k=i,\end{array}\right.$ for $1 \leq$
$k \leq I$. 


$$
\begin{gathered}
\frac{f_{j}}{\mu_{0 k}} \frac{\partial \mu_{0 k}}{\partial f_{j}} \geq\left\{\begin{array}{ll}
\frac{1}{f_{j}^{*}} \frac{f_{k}}{f_{k}^{*}} \sum_{i=1}^{I} \frac{\left[\alpha^{a} \mu_{i k}^{a}+\alpha^{b} \mu_{i k}^{b}\right]\left[\beta^{a} \mu_{i k}^{a}+\beta^{b} \mu_{i k}^{b}\right]}{m_{i}^{*}}>0 & \text { if } k \neq j \\
\frac{f_{j}}{f_{j}^{*}}\left[1+\frac{1}{f_{j}^{*}} \sum_{i=1}^{I} \frac{\left[\alpha^{a} \mu_{i j}^{a}+\alpha^{b} \mu_{i j}^{b}\right]\left[\beta^{a} \mu_{i j}^{a}+\beta^{b} \mu_{i j}^{b}\right]}{m_{i}^{*}}\right]>1 & \text { if } k=j,
\end{array}\right\} \\
k \leq J . \\
\frac{m_{i}}{\mu_{0 j}} \frac{\partial \mu_{0 j}}{\partial m_{i}} \leq-\frac{\left[\alpha^{a} \mu_{i j}^{a}+\alpha^{b} \mu_{i j}^{b}\right]}{m_{i}^{*} f_{j}^{*}} m_{i}<0
\end{gathered}
$$

and

$$
\frac{f_{j}}{\mu_{i 0}} \frac{\partial \mu_{i 0}}{\partial f_{j}} \leq-\frac{\left[\beta^{a} \mu_{i j}^{a}+\beta^{b} \mu_{i j}^{b}\right]}{m_{i}^{*} f_{j}^{*}} f_{j}<0
$$

for $1 \leq i \leq I$ and $1 \leq j \leq J$.

\section{B.3 Proof of Proposition 2}

Recall, from the result of Theorem 1 we know that the fixed point representation (44) admits a unique solution. Therefore, $\mu-B(\mu ; m, f, \theta)$ must be at least locally invertible at the equilibrium. This ensures that it jacobian matrix $J(\mu)$ does not vanish at the equilibrium. Then, $\operatorname{det}(J(\mu)) \neq 0$ for all $\beta^{r}, \alpha^{r}>0$. Since we shown within Step 1 of proof of Theorem 2 that $J(\mu)=U(\mu) H(\mu) U(\mu)^{-1}$ for all $\beta^{r}, \alpha^{r}>0$, we have then $\operatorname{det}(H(\mu)) \neq 0$. Moreover, we have shown that

$\sum_{i=1}^{I} U(\mu)^{-1} \frac{\partial \mu}{\partial m_{i}} m_{i}+\sum_{j=1}^{J} U(\mu)^{-1} \frac{\partial \mu}{\partial f_{j}} f_{j}=\sum_{i=1}^{I}\left[H(\mu)^{-1}\right]_{\cdot i}+\sum_{j=1}^{J}\left[H(\mu)^{-1}\right]_{\cdot(I+j)}$.

If $\beta^{r}+\alpha^{r}=1$, we observe that all elements of $H(\mu)$ are non-negative and the rows sum to one. Therefore, $H(\mu)$ is a row stochastic matrix, see Horn and Johnson (2013, p.547), with an inverse whose rows also sum to one. Then,

$$
\left[H(\mu)^{-1}\right]_{\cdot i}+\sum_{j=1}^{J}\left[H(\mu)^{-1}\right]_{\cdot(I+j)}=\iota_{I+J}
$$

where $\iota_{I+J}=(1, \ldots, 1)^{\prime}$. The last equality holds since the rows of $\left[H(\mu)^{-1}\right]$ sum to one.

\section{Figures and Tables}


Figure 1: Marital Status by Gender and Year.

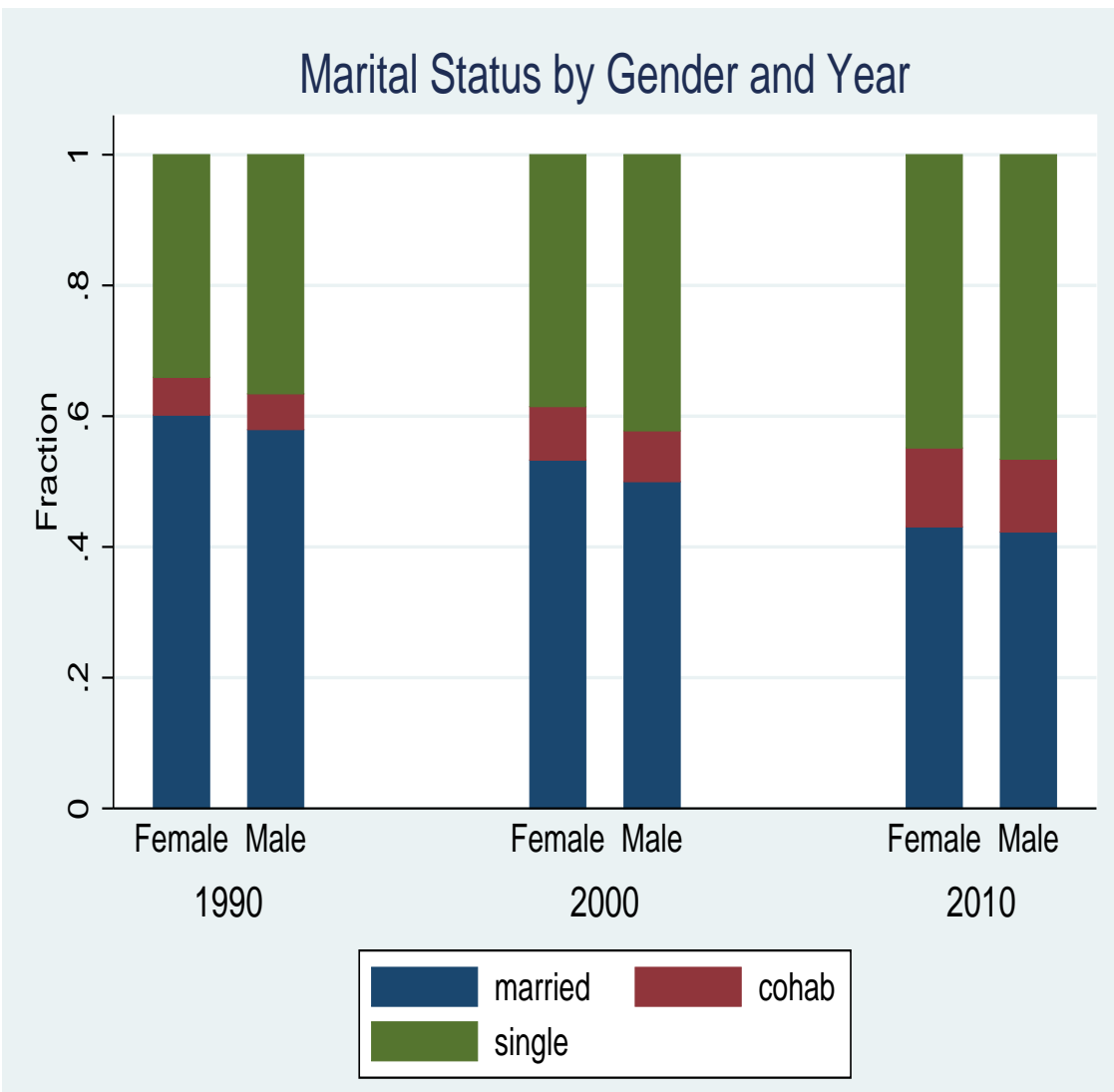


Figure 2: Fraction of individual by gender, education and year.

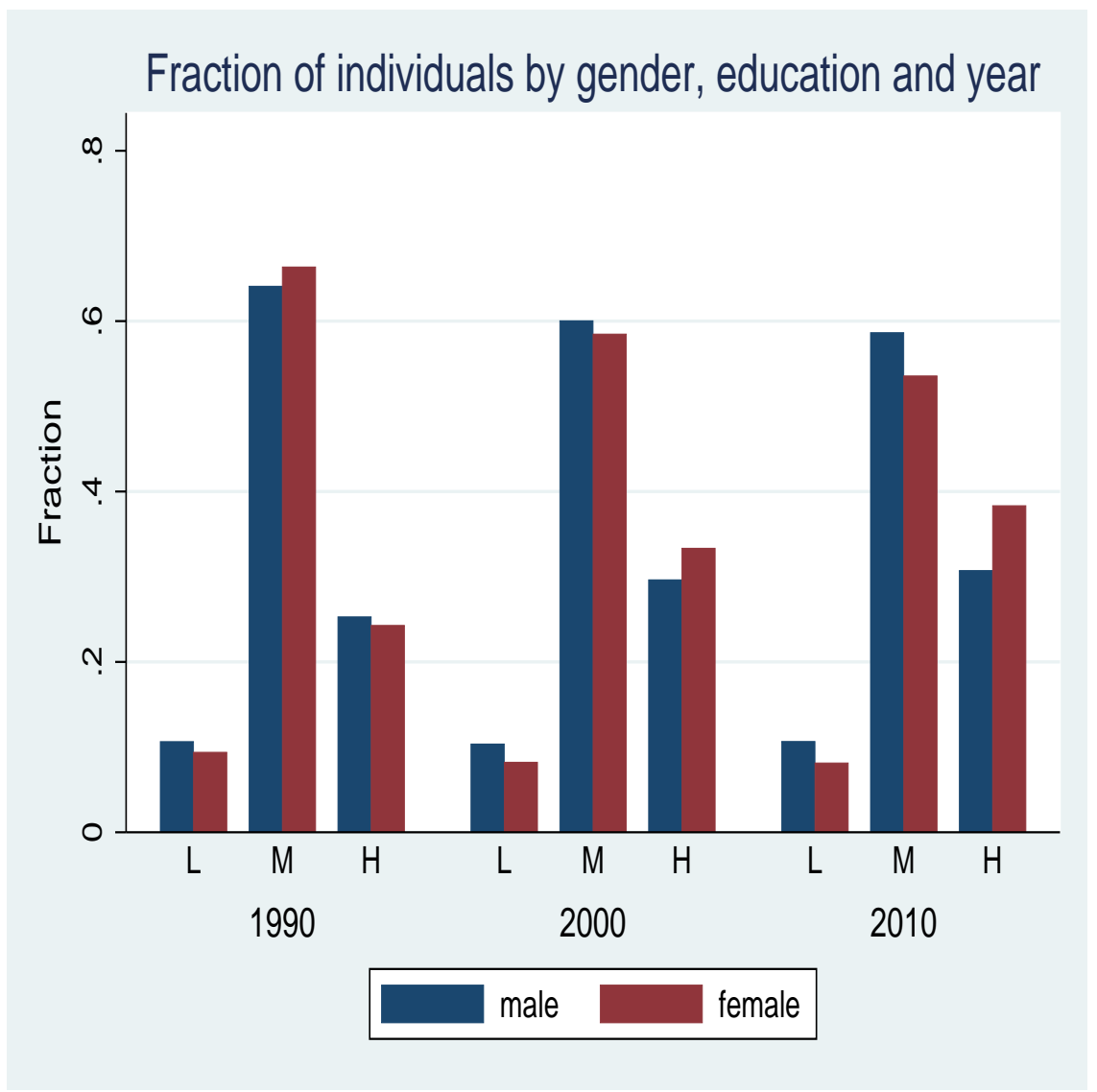


Table 1: Summary Statics*.

\begin{tabular}{|l|l|l|l|l|l|}
\hline Variable & Obs & Mean & Std. Dev. & Min & Max \\
\hline N cohabitations & 1113 & 710.7491 & 1367.211 & 3 & 15362 \\
\hline N marriages & 1283 & 5023.924 & 9981.822 & 6 & 118867 \\
\hline N males & 1377 & 49097.82 & 67662.33 & 174 & 568449 \\
\hline N females & 1377 & 48319.55 & 67411 & 143 & 580493 \\
\hline N unmatched males & 1377 & 20361.38 & 30701.33 & 165 & 262267 \\
\hline N umatched females & 1377 & 18757.32 & 28271.38 & 76 & 236391 \\
\hline Year & 1377 & 2000 & 8.167932 & 1990 & 2010 \\
\hline
\end{tabular}

*An observation is a state/year. There are 51 states which includes DC. Observations with 0 cohabitation or marriages are excluded. 
Table 2: Ordinary Least Square (OLS).

\begin{tabular}{|c|c|c|c|c|c|c|}
\hline & la & $1 b$ & $2 \mathrm{a}$ & $2 b$ & $3 a$ & $3 b$ \\
\hline Dep. Var. & $\mathrm{LCOH}$ & LMAR & $\mathrm{LCOH}$ & LMAR & $\mathrm{LCOH}$ & LMAR \\
\hline LU_M $(\alpha)$ & $\begin{array}{c}0.562 \\
(0.041)^{* *}\end{array}$ & $\begin{array}{c}0.536 \\
(0.048)^{* *}\end{array}$ & $\begin{array}{c}0.320 \\
(0.075)^{* *}\end{array}$ & $\begin{array}{c}0.244 \\
(0.061)^{* *}\end{array}$ & $\begin{array}{c}0.557 \\
(0.084)^{* *}\end{array}$ & $\begin{array}{c}0.626 \\
(0.056)^{* *}\end{array}$ \\
\hline $\begin{array}{l}\text { LU_F }(\beta) \\
L\left[\frac{H H * M M}{H M * M H} .\right.\end{array}$ & $\begin{array}{c}0.531 \\
(0.040)^{* *}\end{array}$ & $\begin{array}{c}0.665 \\
(0.047)^{* *}\end{array}$ & $\begin{array}{c}0.630 \\
(0.076)^{* *} \\
2.31 \\
(0.078)^{* *}\end{array}$ & $\begin{array}{c}0.609 \\
(0.058)^{* *} \\
2.44 \\
(0.069)^{* *}\end{array}$ & $\begin{array}{c}0.827 \\
(0.078)^{* *} \\
2.290 \\
(0.071)^{* *}\end{array}$ & $\begin{array}{c}0.939 \\
(0.051)^{* *} \\
2.412 \\
(0.048)^{* *}\end{array}$ \\
\hline$L\left[\frac{M M * L L}{L M * M L}\right]$ & & & $\begin{array}{c}1.78 \\
(0.087)^{* *}\end{array}$ & $\begin{array}{c}2.52 \\
(0.082)^{* *}\end{array}$ & $\begin{array}{c}1.781 \\
(0.080)^{* *}\end{array}$ & $\begin{array}{c}2.464 \\
(0.063)^{* *}\end{array}$ \\
\hline$L\left[\frac{H M * M L}{M M * H L}\right]$ & & & $\begin{array}{c}0.784 \\
(0.147)^{* *}\end{array}$ & $\begin{array}{c}1.43 \\
(0.092)^{* *}\end{array}$ & $\begin{array}{c}0.796 \\
(0.145)^{* *}\end{array}$ & $\begin{array}{c}1.426 \\
(0.084)^{* *}\end{array}$ \\
\hline$L\left[\frac{M H * L M}{M M * L H}\right]$ & & & $\begin{array}{c}1.33 \\
(0.141)^{* *}\end{array}$ & $\begin{array}{c}1.37 \\
(0.101)^{* *}\end{array}$ & $\begin{array}{c}1.344 \\
(0.135)^{* *}\end{array}$ & $\begin{array}{c}1.379 \\
(0.083)^{* *}\end{array}$ \\
\hline Y2000 & & & $\begin{array}{c}0.289 \\
(0.042)^{* *}\end{array}$ & $\begin{array}{c}-0.287 \\
(0.032)^{* *}\end{array}$ & $\begin{array}{c}0.313 \\
(0.037)^{* *}\end{array}$ & $\begin{array}{c}-0.277 \\
(0.022)^{* *}\end{array}$ \\
\hline Y2010 & & & $\begin{array}{c}0.627 \\
(0.041)^{* *}\end{array}$ & $\begin{array}{c}-0.604 \\
(0.037)^{* *}\end{array}$ & $\begin{array}{c}0.615 \\
(0.040)^{* *}\end{array}$ & $\begin{array}{c}-0.667 \\
(0.030)^{* *}\end{array}$ \\
\hline STATE & & & & & $\mathrm{Y}$ & Y \\
\hline _cons & $\begin{array}{c}-4.788 \\
(0.383)^{* *}\end{array}$ & $\begin{array}{c}-3.981 \\
(0.441)^{* *}\end{array}$ & $\begin{array}{c}-2.842 \\
(0.196)^{* *}\end{array}$ & $\begin{array}{c}1.172 \\
(0.158)^{* *}\end{array}$ & $\begin{array}{c}-7.359 \\
(0.772)^{* *}\end{array}$ & $\begin{array}{c}-5.015 \\
(0.543)^{* *}\end{array}$ \\
\hline$R^{2}$ & 0.51 & 0.45 & 0.90 & 0.95 & 0.92 & 0.97 \\
\hline$N$ & 1,113 & 1,283 & 1,113 & 1,283 & 1,113 & 1,283 \\
\hline $\begin{array}{c}\frac{\alpha}{\beta} \\
\alpha+\beta\end{array}$ & $\begin{array}{c}1.058 \\
(0.137) \\
1.093 \\
(0.039)\end{array}$ & $\begin{array}{c}0.806 \\
(0.115) \\
1.202 \\
(0.045)\end{array}$ & $\begin{array}{c}0.508 \\
(0.178) \\
0.950 \\
(0.018)\end{array}$ & $\begin{array}{c}0.400 \\
(0.138) \\
0.853 \\
(0.016)\end{array}$ & $\begin{array}{c}0.673 \\
(0.146) \\
1.384 \\
(0.079)\end{array}$ & $\begin{array}{c}0.667 \\
(0.082) \\
1.57 \\
(0.057)\end{array}$ \\
\hline$\frac{\alpha^{\mathcal{M}}}{\beta^{\mathcal{M}}} \frac{\beta^{\mathcal{C}}}{\alpha^{\mathcal{C}}}$ & & $\begin{array}{c}0.762 \\
(0.147)\end{array}$ & & $\begin{array}{c}0.788 \\
(0.387)\end{array}$ & & $\begin{array}{c}0.991 \\
(0.246)\end{array}$ \\
\hline $\operatorname{prob}\left[\begin{array}{l}\alpha^{\mathcal{M}} \\
\beta^{\mathcal{M}}\end{array}\right.$ & $\left.\begin{array}{l}\mathcal{l}=\alpha^{\mathcal{C}} \\
\\
=\beta^{\mathcal{C}}\end{array}\right]$ & 0.091 & & 0.001 & & 0.117 \\
\hline
\end{tabular}

${ }^{*} p<0.05 ;{ }^{* *} p<0.01$ 
Table 3: Instrumental Variable (IV).

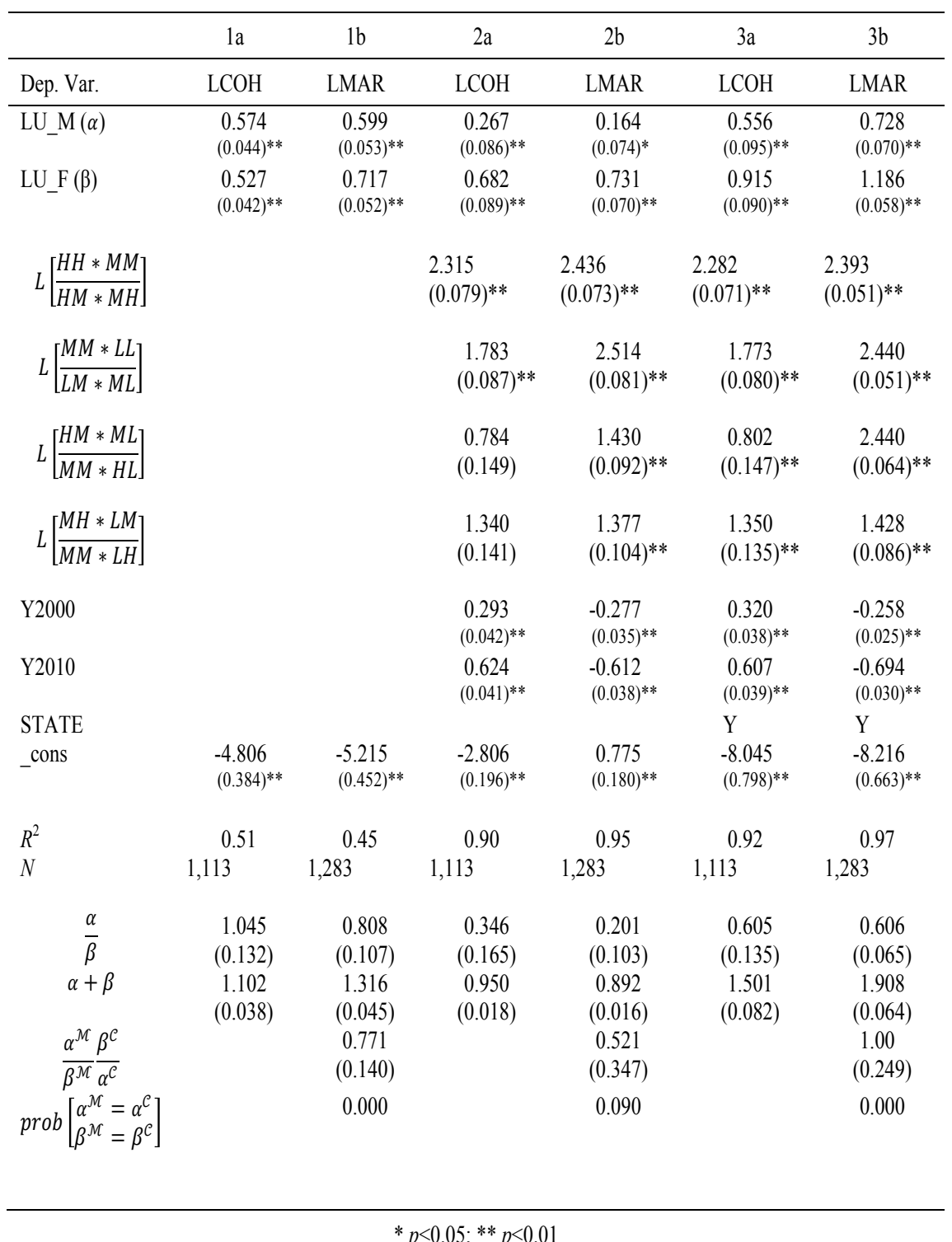


Table 4: IV with time varying match effects.

\begin{tabular}{|c|c|c|c|c|}
\hline & 1a & $1 \mathrm{~b}$ & $2 \mathrm{a}$ & $2 b$ \\
\hline Dependent variable & $\mathrm{LCOH}$ & LMAR & $\mathrm{LCOH}$ & LMAR \\
\hline LU_M $(\alpha)$ & $\begin{array}{l}0.415 \\
(0.071)^{* *}\end{array}$ & $\begin{array}{l}0.357 \\
(0.064)^{* *}\end{array}$ & $\begin{array}{l}0.576 \\
(0.077)^{* *}\end{array}$ & $\begin{array}{l}0.754 \\
(0.057)^{* *}\end{array}$ \\
\hline LU_F $(\beta)$ & $\begin{array}{l}0.528 \\
(0.071)^{* *}\end{array}$ & $\begin{array}{l}0.524 \\
(0.063)^{* *}\end{array}$ & $\begin{array}{l}0.688 \\
(0.073)^{* *}\end{array}$ & $\begin{array}{l}0.885 \\
(0.050)^{* *}\end{array}$ \\
\hline$L\left[\frac{H H * M M}{H M * M H}\right]$ & $\begin{array}{l}2.288 \\
(0.148)^{* *}\end{array}$ & $\begin{array}{l}2.458 \\
(0.087)^{* *}\end{array}$ & $\begin{array}{l}2.278 \\
(0.145) * *\end{array}$ & $\begin{array}{l}2.440 \\
(0.045)^{* *}\end{array}$ \\
\hline$L[M M * L L]$ & 1.504 & 2.255 & 1.514 & 2.198 \\
\hline${ }^{L}\lfloor\overline{L M * M L}\rfloor$ & $(0.116)^{* *}$ & $(0 . .121)^{* *}$ & $(0.103)^{* *}$ & $(0.067)^{* *}$ \\
\hline$L\left[\frac{H M * M L}{M M * H L}\right]$ & $\begin{array}{l}1.169 \\
(0.283)^{* * *}\end{array}$ & $\begin{array}{l}1.698 \\
(0.136)^{* *}\end{array}$ & $\begin{array}{l}0.787 \\
(0.211)^{* *}\end{array}$ & $\begin{array}{l}1.702 \\
(0.110)^{* *}\end{array}$ \\
\hline$L\left[\frac{M H * L M}{M M * L H}\right]$ & $\begin{array}{l}0.693 \\
(0.260)^{* *}\end{array}$ & $\begin{array}{l}1.305 \\
(0.134)^{* *}\end{array}$ & $\begin{array}{l}1.177 \\
(0.266)^{* *}\end{array}$ & $\begin{array}{l}1.313 \\
(0.119)\end{array}$ \\
\hline$L\left[\frac{H H * M M}{H M * M H}\right] * Y 2000$ & $\begin{array}{c}0.259 \\
(0.181)\end{array}$ & $\begin{array}{l}-0.011 \\
(0.191)\end{array}$ & $\begin{array}{l}0.663 \\
(0.230)^{* *}\end{array}$ & $\begin{array}{l}-0.097 \\
(0.119)\end{array}$ \\
\hline$L\left[\frac{M M * L L}{U L}\right] * Y 2000$ & 0.346 & 0.346 & 0.257 & 0.337 \\
\hline$L\left[\frac{H M * M L}{M M * H L}\right] * Y 2000$ & $\begin{array}{c}(0.323) \\
0.032 \\
(0.330)\end{array}$ & $\begin{array}{l}(0.186) \\
-0.279 \\
(0.187)\end{array}$ & $\begin{array}{c}(0.153) \\
0.332 \\
(0.317)\end{array}$ & $\begin{array}{l}(0.124)^{* *} \\
-0.274 \\
(0.151)\end{array}$ \\
\hline$L\left[\frac{M H * L M}{M M * L H}\right] * Y 2000$ & $\begin{array}{c}1.133 \\
(0.252)\end{array}$ & $\begin{array}{l}-0.042 \\
(0.193)\end{array}$ & $\begin{array}{c}0.040 \\
(0.311)\end{array}$ & $\begin{array}{l}-0.052 \\
(0.152)\end{array}$ \\
\hline$L\left[\frac{H H * M M}{H M * M H}\right] * Y 2010$ & $\begin{array}{l}1.133 \\
(0.252)^{* *}\end{array}$ & $\begin{array}{l}-0.208 \\
(0.199)\end{array}$ & $\begin{array}{l}1.062 \\
(0.232)^{* *}\end{array}$ & $\begin{array}{l}-0.396 \\
(0.128)\end{array}$ \\
\hline$L\left[\frac{M M * L L}{U N}\right] * Y 2010$ & 0.692 & 0.534 & 0.679 & 0.540 \\
\hline$L\left[\frac{H M * M L}{M M * H L}\right] * Y 2010$ & $\begin{array}{l}(0.190)^{* *} \\
-0.384 \\
(0.261)\end{array}$ & $\begin{array}{l}(0.200)^{* *} \\
-0.609 \\
(0.268)^{* *}\end{array}$ & $\begin{array}{l}(0.181)^{* *} \\
-0.323 \\
(0.261)\end{array}$ & $\begin{array}{l}(0.162)^{* *} \\
-0.655 \\
(0.246)^{* *}\end{array}$ \\
\hline$L\left[\frac{M H * L M}{M M * L H}\right] * Y 2010$ & $\begin{array}{c}0.394 \\
(0.392)\end{array}$ & $\begin{array}{c}0.264 \\
(0.234)\end{array}$ & $\begin{array}{c}0.391 \\
(0.376)\end{array}$ & $\begin{array}{c}0.237 \\
(0.203)\end{array}$ \\
\hline Y 2000 & $\begin{array}{l}0.693 \\
(0.104)^{* *}\end{array}$ & $\begin{array}{c}0.014 \\
(0.075)\end{array}$ & $\begin{array}{l}0.669 \\
(0.091)^{* *}\end{array}$ & $\begin{array}{l}-0.071 \\
(0.054)\end{array}$ \\
\hline Y2010 & $\begin{array}{l}1.122 \\
(0.102)^{* *}\end{array}$ & $\begin{array}{l}-0.097 \\
(0.079)\end{array}$ & $\begin{array}{l}1.061 \\
(0.092)^{* *}\end{array}$ & $\begin{array}{l}-0.274 \\
(0.057)^{* *}\end{array}$ \\
\hline STATE & & & $\mathrm{Y}$ & $\mathrm{Y}$ \\
\hline _cons & $\begin{array}{l}-3.075 \\
(0.185)^{* *}\end{array}$ & $\begin{array}{l}0.618 \\
(0.161)^{* *}\end{array}$ & $\begin{array}{l}-6.512 \\
(0.735)^{* *}\end{array}$ & $\begin{array}{l}-5.900 \\
(0.506)^{* *}\end{array}$ \\
\hline$R^{2}$ & 0.91 & 0.96 & 0.93 & 0.98 \\
\hline$N$ & 1,113 & 1,283 & 1,113 & 1,283 \\
\hline$\underline{\alpha}$ & 0.786 & 0.681 & 0.836 & 0.852 \\
\hline $\bar{\beta}$ & $(0.239)$ & $(0.203)$ & $(0.174)$ & $(0.096)$ \\
\hline$\alpha+\beta$ & $\begin{array}{c}0.943 \\
(0.017)\end{array}$ & $\begin{array}{c}0.881 \\
(0.016)\end{array}$ & 1.264 & $\begin{array}{l}1.640 \\
(0.055)\end{array}$ \\
\hline$\frac{\alpha^{\mathcal{M}}}{\beta^{\mathcal{M}}} \frac{\beta^{\mathcal{C}}}{\alpha^{\mathcal{C}}}$ & $(0.017)$ & $\begin{array}{c}(0.010) \\
0.866 \\
(0.369)\end{array}$ & $(0.0 / 0)$ & $\begin{array}{l}1.019 \\
(0.241)\end{array}$ \\
\hline $\operatorname{prob}\left[\begin{array}{l}\alpha^{\mathcal{M}}=\alpha^{\mathcal{C}} \\
\beta^{\mathcal{M}}=\beta^{\mathcal{C}}\end{array}\right]$ & & 0.051 & & 0.000 \\
\hline
\end{tabular}

